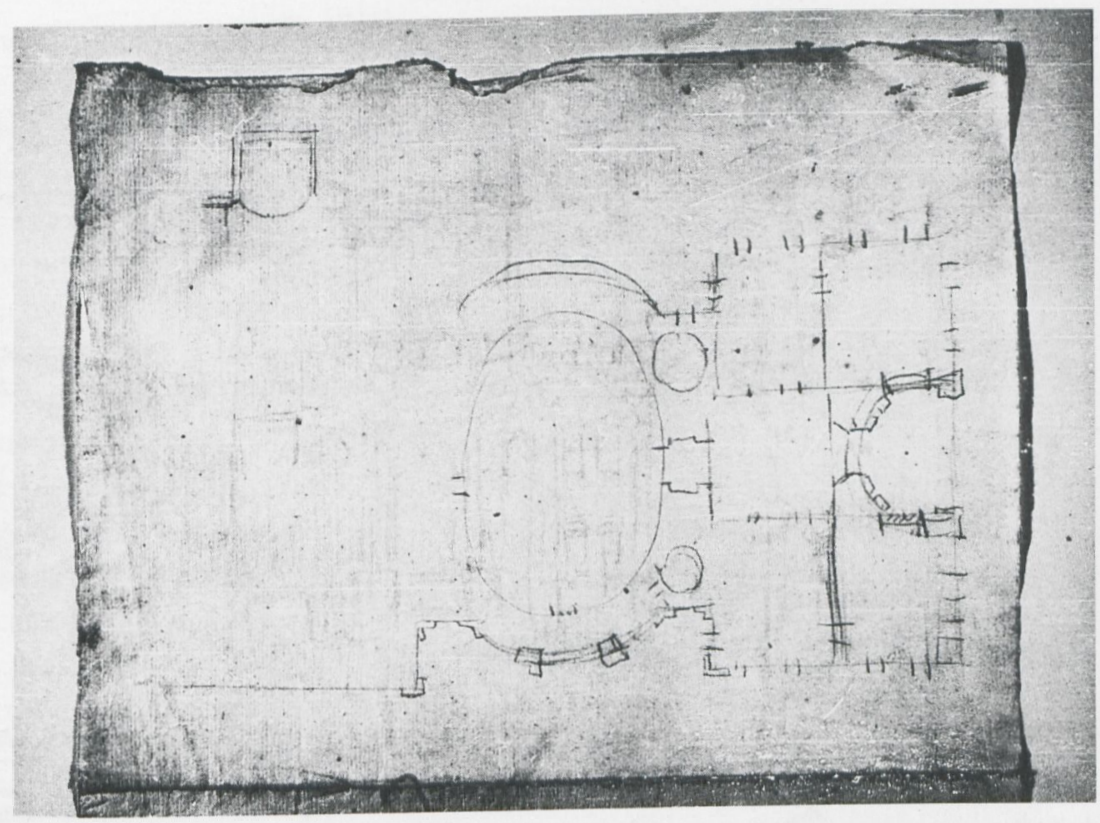

I. Grundriß des Lustgartengebäudes, Warschau, Univ.-Bibl. Arch.

Tilm. 462 recto-C

Stanisław Mossakowski

\title{
Zwischen Rom, München und Warschau. Rätselhafte Zeichnungen in der Sammlung des Architekten Tilman van Gameren
}

Dem Andenken von Jan Białostocki gewidmet

In dem umfangreichen, über neunhundert Blätter mit Skizzen und zeichnerischen Entwürfen umfassenden Nachlaß des hervorragenden Architekten Tilman van Gameren - eines um I630 geborenen, in Italien ausgebildeten, später polonisierten Holländers, der von $166 \mathrm{r}$ bis zu seinem Tode im

(Übersetzung aus dem Polnischen von Juliane Marquard-Twarowski)

Der vorliegende Artikel wurde am I6. Dezember I991 auf der Konferenz $»$ Die kulturelle Dimension Europas am Beispiel der Graubündner Baumeister und Stukkateure« in Traunstein vorgetragen.

${ }^{\text {I }}$ Sie unterscheiden sich von Tilmans Zeichnungen vor allem durch eine andere Technik - van Gameren meidet z. B. perspektivische Aufnahmen - und auch durch das
Jahr 1706 in Polen gearbeitet hat - bilden Zeichnungen anderer Architekten eine zahlenmäßig kleine Gruppe ${ }^{1}$. Unter diesen haben die drei Skizzen, die auf dem Blatt AT 462 recto und verso sichtbar sind, die besondere Aufmerksamkeit der Wissenschaftler erregt (Abb. I, 4, 6) 2 . Es handelt

Fehlen der für den Holländer charakteristischen Meisterschaft der Skizzierung bildhauerischer Details. Vgl. Stanisław Mossakowski: Tilman van Gameren. Leben und Werk, München 1994 .

${ }^{2}$ Warschau, Gabinet Rycin Biblioteki Uniwersyteckiej, Archiwum Tylmana, AT 462, Bleistift, Maße: 395 x 270 $\mathrm{mm}$. Diese und andere Zeichnungen aus der hier besprochenen Sammlung stammen aus der Zeit vor 1706, nach van Gamerens Tod wurden die Zeichnungen gemäß seinem letzten Willen der Bibliothek des Kapuzi- 


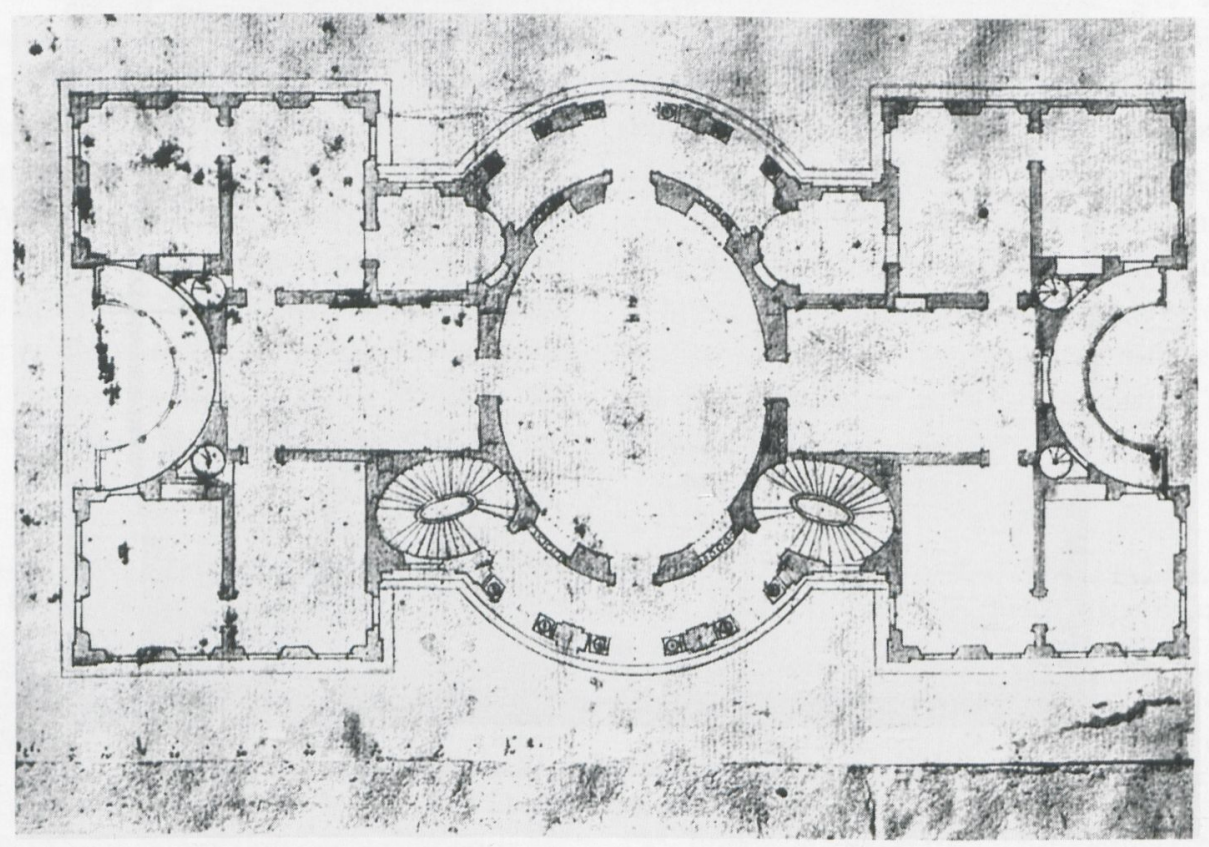

2. J. B. Fischer von Erlach, Grundriß des Lustgartengebäudes, Wien, Albertina, Cod. Montenuovo, f. I6 recto

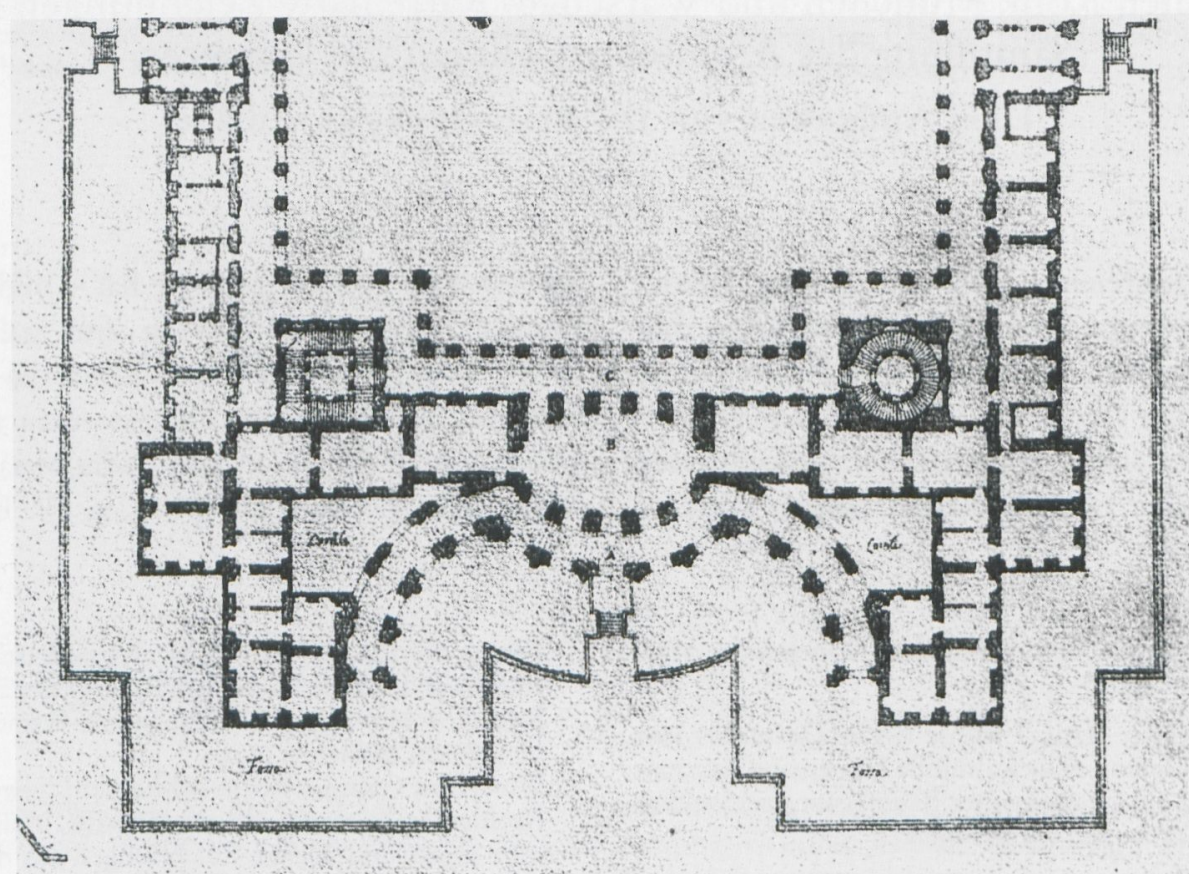

3. G. L. Bernini, Ovalplan für den Louvre, Grundriß, Paris, Cabinet des Estampes, Band: »Palais du Louvre«, Bd. I. f. 3 - Fragment 
sich um Studien für den Grundriß, die Haupt- und Seitenfassade sowie den Querschnitt eines Gartenschlößchens, wie wir es von zahlreichen Entwürfen Johann Bernhard Fischers von Erlach kennen und wie es unter der Bezeichnung » Fischers Lustgartengebäude« von Hans Sedlmayr als die "glanzvollste Invention im gesamten frühen $\mathrm{Eu}-$ vre« des österreichischen Meisters in die Architekturgeschichte eingegangen ist (Abb. 2$)^{3}$.

$\mathrm{Da}$ B Berninis berühmter erster Louvre-Entwurf aus dem Jahre r664 das Vorbild für die Konzeption "Lustgartengebäude« war, ist seit langem bekannt ${ }^{4}$. Jedoch brachten erst die Forschungen von Hellmut Lorenz und Joseph Connors ans Licht, daß Fischers Entwürfe direkt an die Ideen nicht identifizierter italienischer Künstler aus dem Bernini-Kreis anknüpften, die wir durch drei Zeichnungen in New York, Edinburgh und Berlin kennen (Abb. 5) 5 . Die beiden letzteren mit dem Entwurf für die Fassade und die Seitenansicht hat Lorenz auf die Zeit um I680 - r684 datiert und mit den Künstlern aus dem Umkreis der römischen Accademia di San Luca verbunden ${ }^{6}$. Bei der ersten Zeichnung, die eine Fassade, geschmückt durch eine Wappenkartusche mit päpstlicher Tiara, darstellt, kann es sich - so vermutet Connors - um eine frühe Entwurfsphase des Chigi-Palastes (spä-

nerklosters in Warschau übergeben, von wo sie schließlich - nach ${ }_{1863}$ - in die Universitätsbibliothek gelangten. Die Skizzen auf dem Blatt AT 462 wurden von Hallström erwähnt, der eine gesonderte Publikation darüber angekündigt hat: Björn Henrik Hallström: Der Baumeister Andreas Schlüter und seine Nachfolge in St. Petersburg, in: Konsthistorisk Tidskrift, 3/4, 196I, I26, Anm. 85; sowie von Věra Naňkova: G. B. Allipran$\mathrm{di}$, architetto di Laino in Valle Intelvi, in: Arte Lombarda, XI, 2, 1966, 142, Anm. 33; ausführlicher hat sie besprochen Hellmut Lorenz: Das "Lustgartengebäude « Fischers von Erlach - Variationen eines architektonischen Themas, in: Wiener Jahrbuch für Kunstgeschichte, XXXII, 1979, 66-68, Abb. 74-75 (im folgenden zitiert als "Lustgartengebäude« I).

${ }^{3}$ Hans Sedlmayr: Johann Bernhard Fischer von Erlach, 2. Aufl., Wien 1976, 93 (im folgenden zitiert: Fischer von Erlach, 1976).

${ }^{4}$ U. a. Hans Sedlmayr: Fischer von Erlach und Bernini, in: Das Münster, 1952, 265-273. Siehe Heinrich Brauer, Rudolf Wittkower: Die Zeichnungen des Gianlorenzo Bernini, Berlin 1931, I29-133, Taf. I75 (Grundriß); Louis Hautecœur: Le Louvre et les Tuileries de Louis XIV,

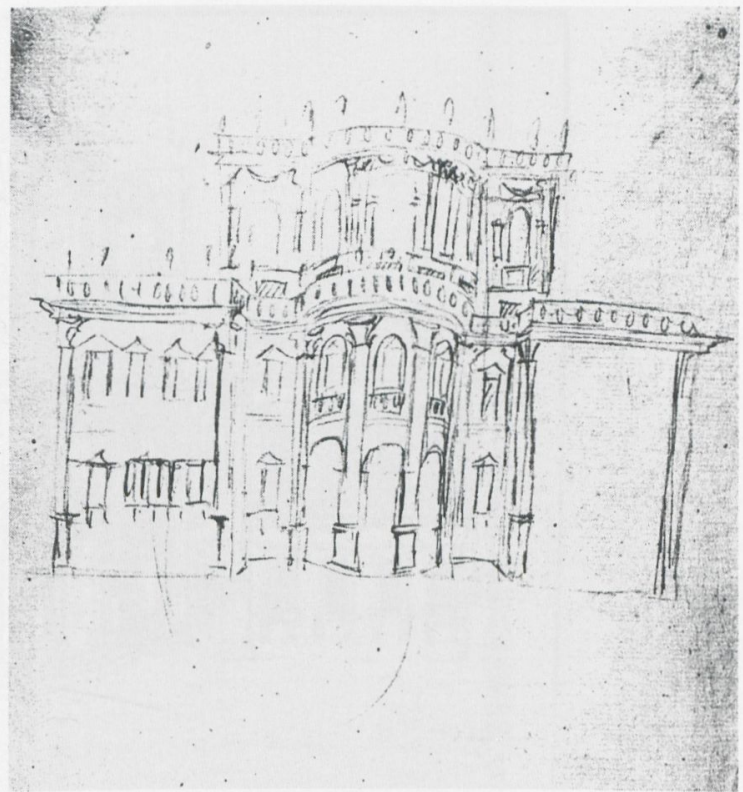

4. Perspektivansicht des Lustgartengebäudes, Warschau, Univ.-Bibl., AT 462 verso-A

ter Odescalchi) handeln, die aus der Zeit um I665 stammt?

Was hatte sich nun der Autor der in Warschau aufbewahrten Skizzen zum Vorbild genommen, wer war er und wie gelangten die Zeichnungen in

Paris 1927, Taf. 33 (Ostfassade); Anthony Blunt: Art and Architecture in France 1500 to 1700, Harmondsworth 1957, Taf. ${ }_{155} \mathrm{~B}$ (andere Zeichnung derselben Fassade).

5 New York, The Cooper-Hewitt Museum, Nr. 1938-883772 (Fassade); Edinburgh, National Gallery of Scotland, Department of Prints and Drawings, ohne Sign. (Fassade); Berlin, Kunstbibliothek, Hdz. II63 (Seitenansicht). Die Zeichnungen aus Edinburgh und Berlin stammen von einer Hand, siehe Hellmut Lorenz: Eine weitere Zeichnung zu Fischers "Lustgartengebäude", in: Wiener Jahrbuch für Kunstgeschichte, XXXIII, I980, 174-I76, Abb. I68-I69 (im folgenden zitiert: "Lustgartengebäude« II).

${ }^{6}$ Lorenz: "Lustgartengebäude« I, 60, 62-63; idem., »Lustgartengebäude « II, 174-176.

${ }^{7}$ Joseph Connors: Bernini's S. Andrea al Quirinale. Payments and Planning, in: Journal of the Society of Architectural Historians, XLI, I, I982, 33-36, Abb. 3I-33. Vgl. Hellmut Lorenz: Bernini e l'architettura barocca austriaca, in: Gian Lorenzo Bernini architetto e l'architettura europea del Sei-Settecento, a cura di G. F. Spagnesi e M. Fagiolo, II, Rom 1984, 655, Anm. 28. 


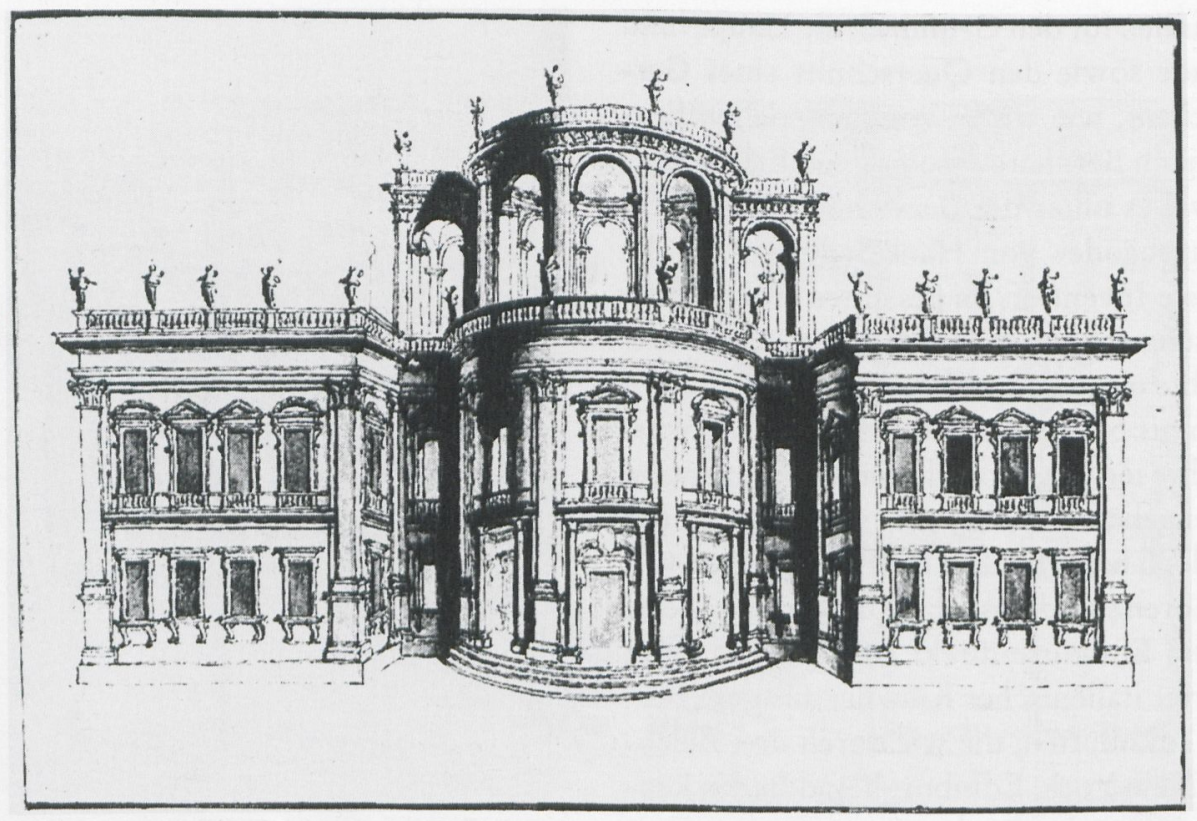

5. Römisch, Frontalansicht des Lustgartengebäudes, Edinburgh, National Gallery of Scotland

die Sammlung Tilmans van Gameren? Das sind einige Fragen, auf die wir eine Antwort zu finden versuchen. Einleitend muß man Hellmut Lorenz zustimmen, der meint: "Trotz der skizzenhaften Flüchtigkeit wirken diese Zeichnungen weniger als spontane Notierung eigener Formideen, sondern sind $\mathrm{m}$. E. eher als rasche... Kopien nach anderen Zeichnungen anzusehen $\ll^{8}$. Ebenso hat Lorenz recht, wenn er eine Einwirkung der Formen von Schloß Liblitz (Liblice bei Melnik), das der Italiener Giovanni Battista Alliprandi, beeinflußt durch das Vorbild Fischers von Erlach ab 1699 errichtet hat, ausschließt, obwohl der Querschnitt auf der Warschauer Skizze die Eindachung des den Bau bekrönenden Geschosses genau so vorsieht, wie es im tschechischen Schloß ausgeführt worden ist (Abb. 6) ${ }^{9}$. Weniger überzeugend erscheint hingegen die Hauptthese von Lorenz,

${ }^{8}$ Lorenz: »Lustgartengebäude I, 67.

${ }^{9}$ Lorenz: »Lustgartengebäude« I, 66-67. Zum Schloß Liblitz siehe: Naňkova (wie Anm. 2), 136-138.

${ }^{10}$ Zagreb, Universitätsbibliothek, Nr. 70-7I (zwei Varianten des Fassadenentwurfs). Siehe Sedlmayr: Fischer der anonyme Autor habe seine Entwurfsskizze in Anlehnung an ihm bekannte Entwurfszeichnungen von Fischer von Erlach bearbeitet, welche zu einer Gruppe von vorbereitenden Studien für den »Entwurf einer Historischen Architectur« (Wien 172I) gehörten und vor 1712, wahrscheinlich um 1705, entstanden sind; sie befinden sich gegenwärtig in der Universitätsbibliothek von Zagreb ${ }^{\text {10 }}$. Trotz der Parallelen in den kompositorischen und dekorativen Details, besonders des Belvedere-Geschosses, das hier wie in Warschau, eine eigene Sockelzone mit Fenstern zur Beleuchtung des Saales im Piano nobile erhielt, sowie auch der Girlanden zwischen den Pilasterkapitellen, ist die Beziehung der dargestellten Bauten keinesfalls so offensichtlich. Ähnlich verhält es sich mit den scheinbar auffälligen Übereinstimmungen zwischen dem Grundriß auf der Warschauer Zeichnung und dem

von Erlach (wie Anm. 3), 305, Abb. 105; Lorenz: »Lustgartengebäude« I, 66-68, Abb. 73, 77.

"Wien, Graphische Sammlung Albertina, "Codex Montenuovo«, Inv. 26.392, fol. 16 recto (Grundriß), Is verso (halbe Fassadenansicht). Siehe: Sedlmayr: Fischer von 


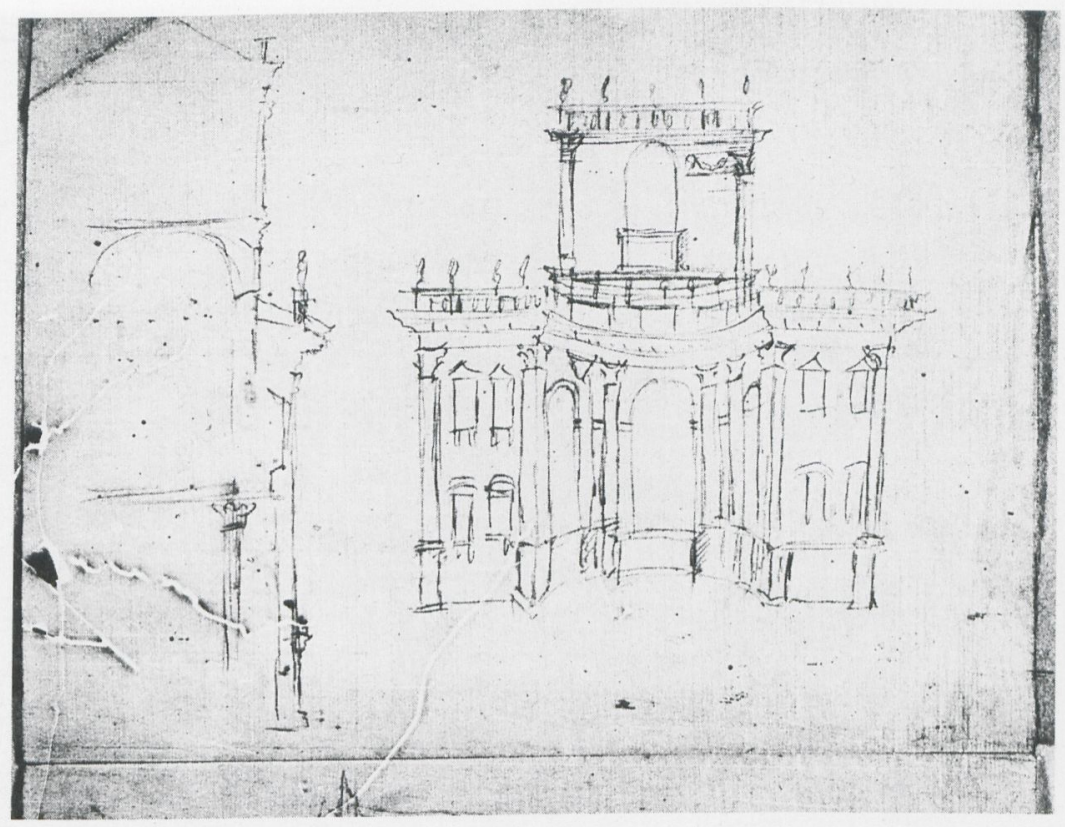

6. Seitenansicht und Querschnitt des Gartenpalais, Warschau, Univ.-Bibl., AT 462 verso-B

anderen auf einer der zwei Fischer von ErlachZeichnungen im Wiener "Codex Montenuovo« (um 1695, Abb. I - 2) II $^{\text {I }}$

Diese und andere Entwürfe für ein »Lustgartengebäude «, angefangen mit dem ältesten aus den Jahren 1687-1690, der - wie Lorenz aufgezeigt hat - für das Gartenpalais des Fürsten Liechtenstein in der Rossau bei Wien bestimmt war, unterscheiden von dem skizzenhaften Projekt in Warschau nämlich eine Reihe wichtiger Elemente ${ }^{12}$. Die Projekte Fischers sehen im Sockelgeschoß vor allem Architrav-Loggien auf Säulenpaaren vor, während der Warschauer Entwurf an dieser Stelle Arkaden einführt. Letztere heben die Warschauer Zeichnung auch von den erwähnten italienischen Entwürfen ab, die den Wiener Architekten inspiriert haben. Sie bilden noch einmal eine deutliche Anknüpfung an die Arkadengalerien des Urbildes - Berninis

Erlach (wie Anm. 3), 314, Abb. 219; Lorenz: "Lustgartengebäude« I, 63-66, Abb. 67-68.

${ }^{12}$ Milano, Castel Sforzesco, Civiche Raccolte d'Arte, Gabinetto dei disegni, Collezione Martinelli, Bd. IX, fol. 33, 23, 25. Siehe: Lorenz: "Lustgartengebäude« I,
Louvre-Projekt. Auch sind die Flankenbauten anders als bei Fischer gestaltet (vgl. hierzu den Grundriß im Codex Montenuovo, fol. I6 recto, Abb. 2). Die drei großen Arkaden im mittleren Rückschwung, die beide Geschosse des Baues überfangen, enthalten, wie der unten sichtbare durchlaufende Sockel bezeugt, keine Eingänge (Abb. 6). Gleichzeitig ergibt die Analyse des Warschauer Grundrisses, daß man die seitlichen Arkaden in Wirklichkeit als dekorative Blendnischen plante und nur die Öffnung der mittleren Arkade vorgesehen war: durch sie sollte Licht in den kleinen rechteckigen Hof fallen, der die Verbindung zwischen den Appartements des Sockelsgeschosses und der ovalen Sala terrena in der Mitte bildet (Abb. I) $)^{13}$. Auch diese kleinen Höfe im Gebäudeinneren sind ein Reflex auf den erwähnten Bernini-Entwurf (Abb. 3).

59-63, 73-74, Abb. 6r, 66, 65; idem: (wie Anm. 7), 650, 655 .

${ }^{13}$ Wir haben es hier auch nicht mit »bloßen Mißverständnissen « des Autors (Kopisten) zu tun, wie Lorenz behauptet ("Lustgartengebäude« I, 67). 


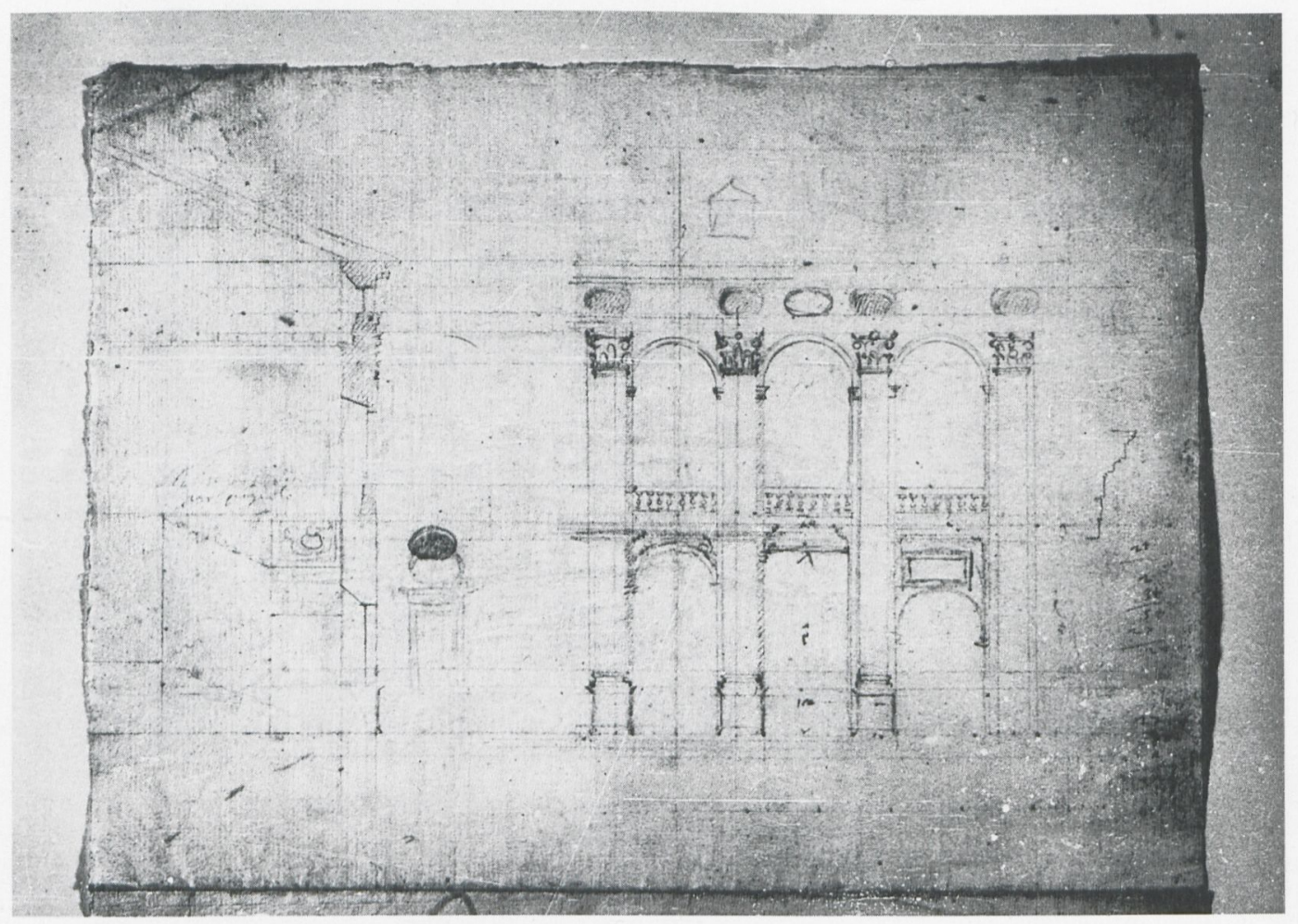

7. Teilansicht und Querschnitt des Gartenpalais, Warschau, Univ.-Bibl., AT 462 recto-D

Somit zwingt nichts zu der Annahme von Lorenz' These, dem Autor der Warschauer Zeichnungen seien die Entwürfe Fischers von Erlach bekannt gewesen. Daß der Italiener bei der Ausführung seiner Skizzen einen anderen italienischen Entwurf vor Augen gehabt hat, der zwischen Berninis Louvre-Konzept und den Entwürfen steht, die sich auf den heute in New York, Edinburg und Berlin aufbewahrten Zeichnungen befinden, kann man hingegen nicht ausschließen.

Den Versuch, die Frage nach dem Autor der Warschauer Skizzen zu beantworten, können weitere Zeichnungen erleichtern: zum einen die

${ }^{14}$ AT 452 - Grundriß- und Fassadenstudie, Bleistift, mit Tusche laviert, Maße: 4 OI X $272 \mathrm{~mm}$.

${ }^{15}$ AT 650 - zwei Portalentwürfe, Bleistift, mit Tusche laviert, Maße 263 x $394 \mathrm{~mm}$; AT 913 recto und verso, Skizzen architektonischer Elemente (Einfassungen von Fenstern bzw. Portalen, vom Gebälk) sowie von Dekorationsdetails, Feder, 271 x 205 mm; AT 46I-Grundriß vierte, genauer ausgearbeitete Zeichnung auf dem schon erwähnten Blatt AT 462 recto, die eine Vorstudie für den Palastentwurf auf der Zeichnung AT 452 vorstellt (Abb. 7,8$)^{14}$, zum anderen einige andere Zeichnungen und Skizzen aus Tilmans Sammlung auf den Blättern AT 650, AT 913 und AT $46 \mathrm{I}^{\mathrm{I}}$. Sie alle weisen die Hand desselben Künstlers auf und sind auf Papier ausgeführt, dessen Wasserzeichen die Form eines sechsarmigen, in einen kreuzbekrönten Kreis eingeschriebenen Sterns hat. Dieses Zeichen unterscheidet sich auf den einzelnen Blättern einzig durch unterschiedliche Majuskeln (R, G) unten am Kreis (Abb. Io $)^{16}$.

und Querschnittstudie zu einem sakralen Zentralbau über dem Grundriß eines Achtecks, das zur Form eines in ein Quadrat eingeschriebenen griechischen Kreuzes ergänzt ist, Bleistift, $264 \times 387 \mathrm{~mm}$.

${ }^{16}$ Buchstabe R - Blatt AT 462; Buchstabe G-Blatt AT $45^{2}$ und 650; Buchstabe F-Blatt AT 913 und 46I. 


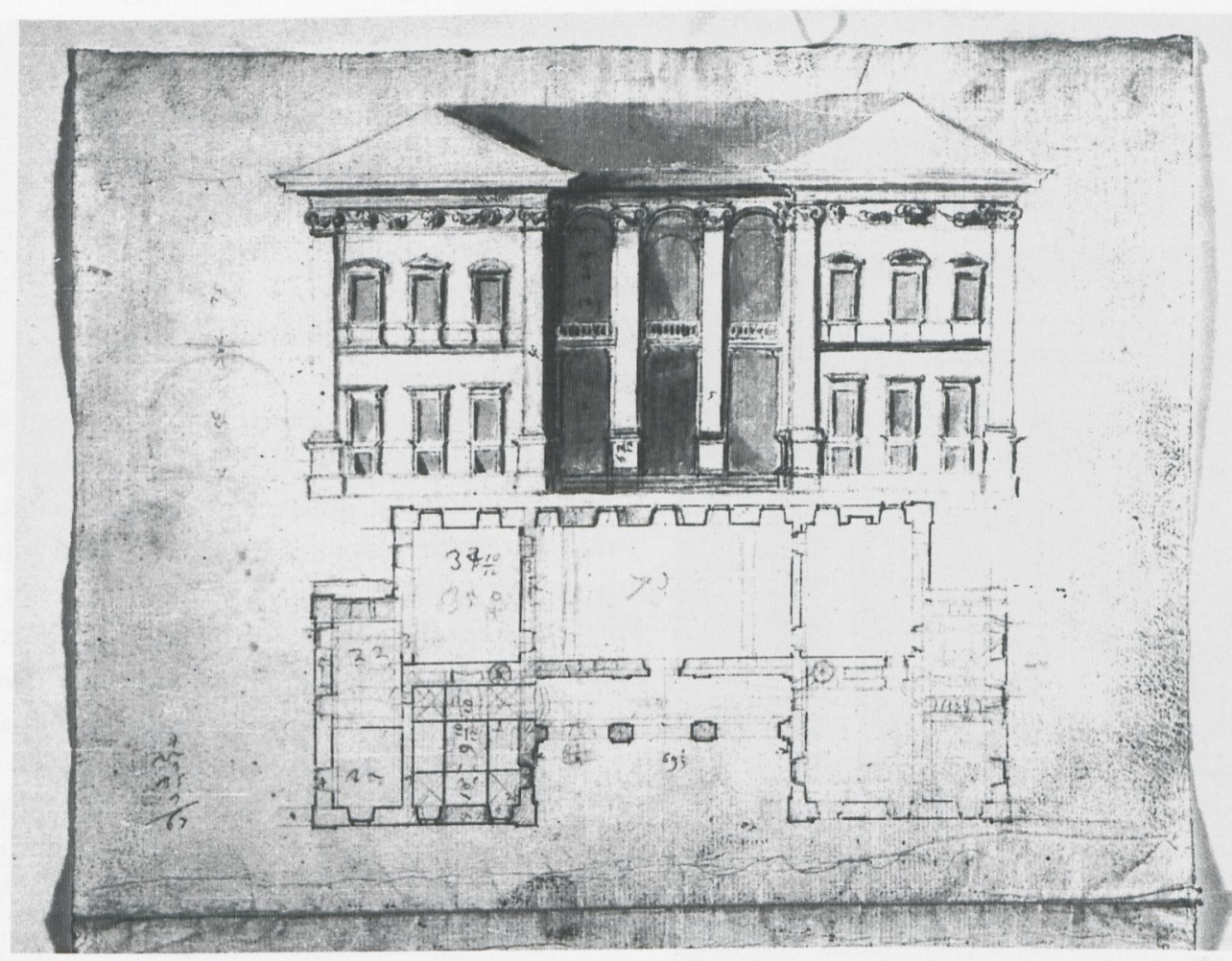

8. Frontalansicht und Grundriß des Gartenpalais, Warschau, Univ.-Bibl., AT 452

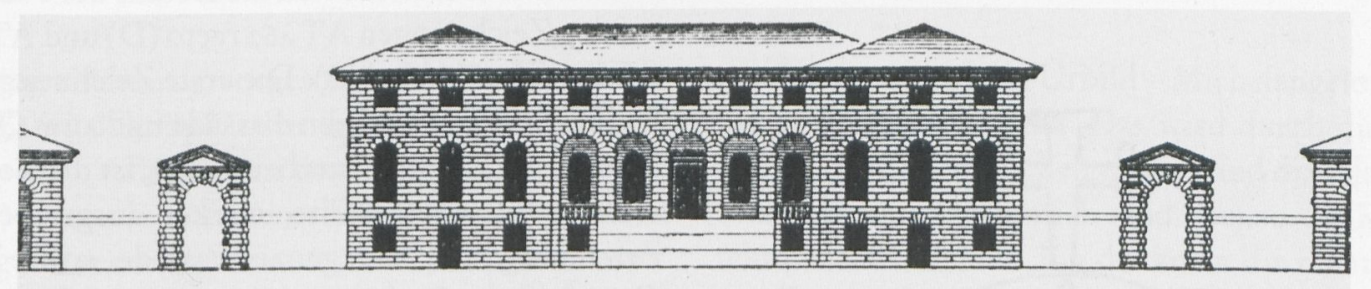

Prospette lemerale,

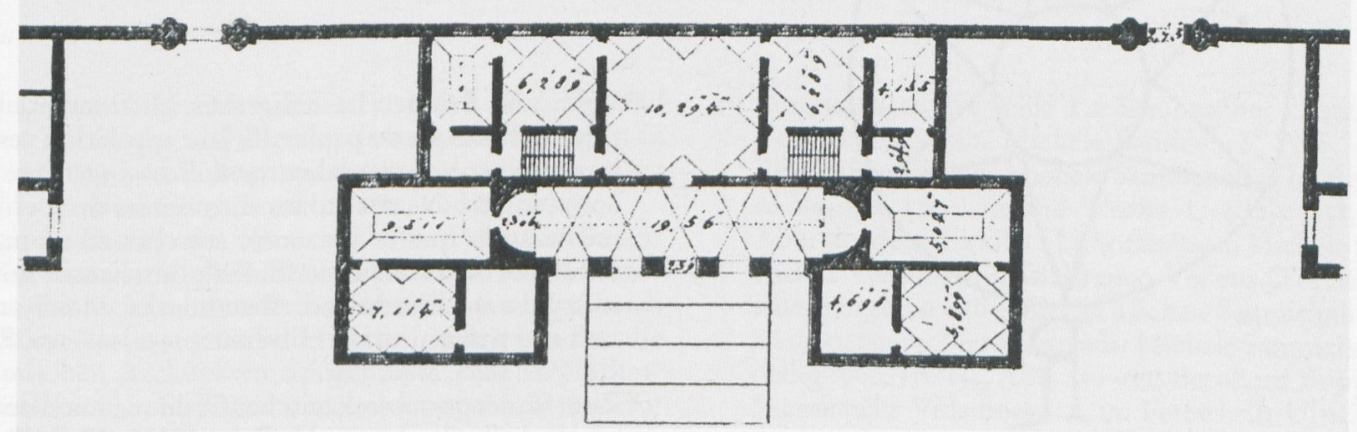

9. M. Sanmicheli, Schloß La Soranza 


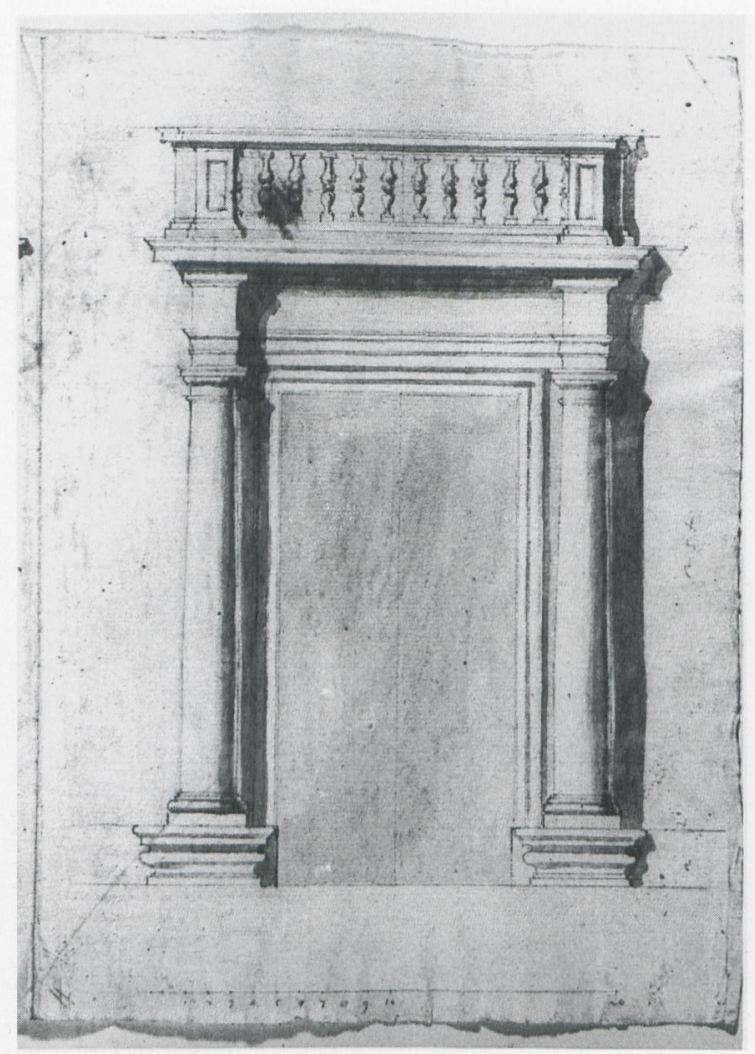

II. Entwurf des Portals, Warschau, Univ.-Bibl., AT 650-B

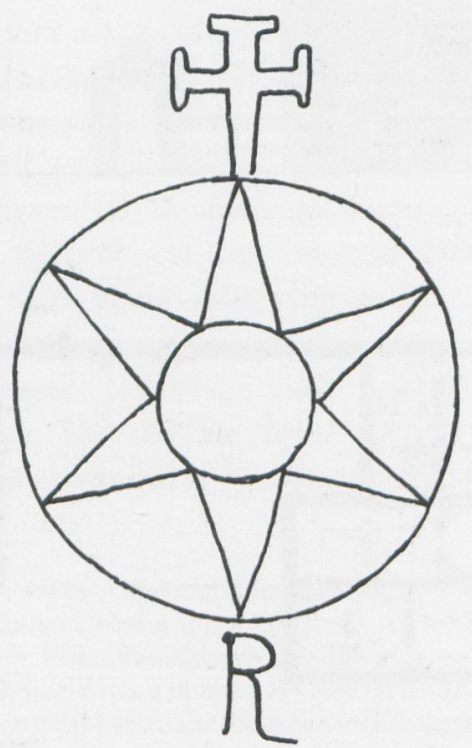

10. Wasserzeichen von Blatt $\mathrm{AT}_{462}$
Ähnliche Wasserzeichen - identische konnten nicht gefunden werden - erscheinen auf Papier, das in verschiedenen großen italienischen Städten z. B. in Rom, Neapel und Lucca ab der Mitte des 16. Jahrhunderts und das ganze 17. Jahrhundert hindurch hergestellt worden ist ${ }^{17}$. Gleichzeitig ist keine der erhaltenen, eigenhändigen TilmanZeichnungen auf Papier mit einem solchen Wasserzeichen ausgeführt worden. Somit handelt es sich zweifellos um die Zeichnungen eines anderen, höchstwahrscheinlich italienischen Künstlers.

Seine italienische, genauer römische Ausbildung wird durch die Entwurfsstudien auf den hier besprochenen Blättern bestätigt. So treffen wir z. B. die Formen der zwei Portale auf der Zeichnung AT 650 (Abb. II) an römischen Palästen wieder, die Ende des 16. und im 17. Jahrhundert entstanden $\operatorname{sind}^{18}$. Dasselbe betrifft die charakteristische Konzeption, die Mezzaninfenster in einem breiten Gebälkfries zwischen Paaren von Volutenkonsolen anzulegen, wie wir es auf der Zeichnung AT 913 recto sehen (Abb. I2) ${ }^{19}$. Die Verbindungen mit Römischem werden hier schließlich durch die daneben angegebene Skizze betont, die eine mit Papsttiara bekrönte Wappenkartusche zeigt.

Vor allem aber leiten sich die Details des Palastes auf den Zeichnungen $\mathrm{AT}_{4} 62$ recto (D) und $\mathrm{AT}_{452}$ aus Rom her (Abb. 7, 8). Die erste Zeichnung, die Studien zu einer Loggien-Fassade und zum Querschnitt mit Treppenhaus beinhaltet, ist die Vorbereitung für die zweite, stärker ausgearbeitete Zeichnung, die die ganze Fassade sowie den Grundriß des Sockelgeschosses mit Maßangaben zu den Räumen zeigt. Die Komposition des Baukörpers mit der eingezogenen Loggia in der Mitte,

${ }^{17}$ Vgl. C. M. Briquet: Les Filigranes. Dictionnaire historique des marque du papier dès leur apparition vers 1282 jusqu'en 1600, Amsterdam 1968, Bd. I, 353, Pos. 6088, 6089 (»on trouve des étoiles surmontées de la croix du même style que le no. 6089, souvent accompagnées d'initiales dans l'Italie méridionale durant tout le XVIIe s.«); Edward Headwood: Watermarks. Mainly of the 17th and I8th centuries, Hilversum 1950, I48-150, Pl. 5155I 6, Pos. 3874, 3880, 3885.

${ }^{18}$ Zum Säulenportal toskanischer Ordnung mit dem Balustradenbalkon vgl. u. a. den Palast Alessandrini-Bonelli, della Prefettura (seit 1585, D. Paganelli, D. Fontana) und 


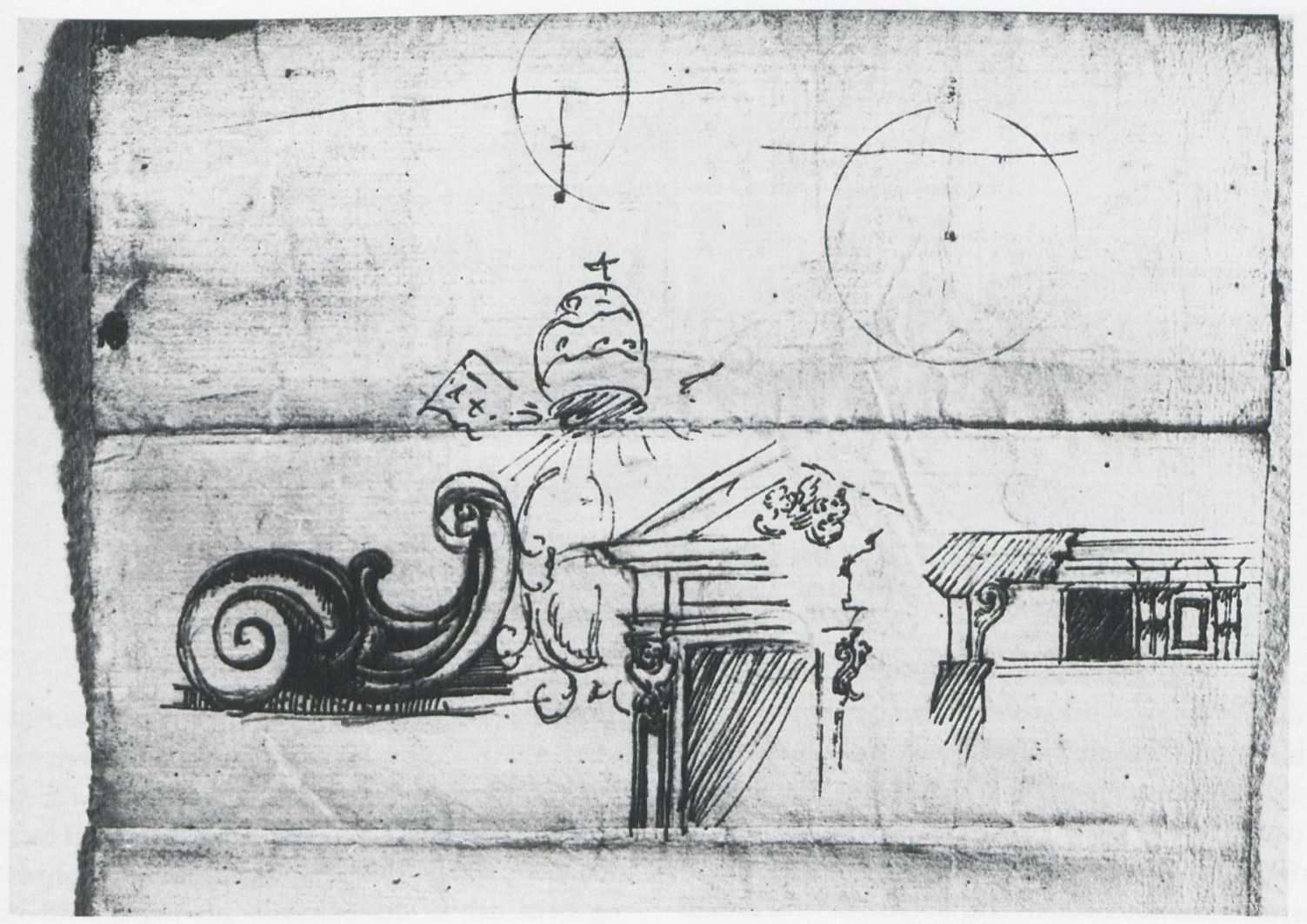

12. Skizzen der architektonischen Details, Warschau, Univ.-Bibl., AT 913 recto

die von risalitartig vorgeschobenen Pavillons eingefaßt wird, knüpft zwar deutlich an die norditalienischen Villenbauten des 16. Jahrhunderts $\mathrm{n}^{20}$. Die kolossale Pilasterordnung (Kompositordnung in der ersten Variante, bzw. jonische Pilaster auf der zweiten Zeichnung) aber zeugt nicht so sehr von der Kenntnis der Werke Palladios (Palazzo Valmarana in Vicenza, 1565-1566), als daß sie

den Palast Chigi-Odescalchi (seit 1664, L. Bernini), und zum Portal, das von Pseudopilastern mit Kapitellen in Form massiver Voluten gerahmt wird, vgl. den Palast Aldobrandini-Chigi (Portal ab 162I, C. Maderno).

19 Vgl. die Fassade des oben erwähnten Palastes Alessandrini-Bonelli. Schon B. Peruzzi hatte in der Villa Farnesina die Mezzaninfenster im Fries des Kranzgesimses plaziert (I506-I5II), und eine solche Lösung wandten die römischen Architekten das ganze 16. und 17. Jh. hindurch an, u. a. D. Fontana im Lateranspalast (1585-1589) sowie L. Bernini bzw. C. Fontana im Palazzo Ludovisi di Montecitorio (1650-1694 und ab 1694). den Rückgriff auf das Urbild - Michelangelos Kapitols-Paläste - beweist. Das wird durch die Architrav-Loggia im Sockelgeschoß und die Pilastersockel bestätigt. Römisch sind ebenso die architektonischen Details. Ich denke an die rechteckigen Fenster des Erdgeschosses, die auf charakteristische Art durch eine gemeinsame Einfassung mit den Fenstern des Sockels verbunden sind, oder an

${ }^{20}$ Besonders an die Villa La Soranza bei Castelfranco Veneto (1540-1550, Michele Sanmicheli, Abb. 9), vgl. Eric Langenskiöld: Michele Sanmicheli. The Architect of Verona. His Life and Works, Uppsala 1938, 90-93, Abb. 36; idem: La villa »La Soranza « di Michele Sanmicheli a Treville di Castelfranco Veneto (Treviso) e lo stile veneziano delle ville, in: Michele Sanmicheli, Verona 1960, 69-73; Piero Gazzola: Michele Sanmicheli, Venedig 1960, I53-I54, Abb. I30-132; Bernhard Rupprecht: Sanmichelis Villa Soranza, in: Festschrift Ulrich Middeldorf, Berlin 1968, 324-332. 


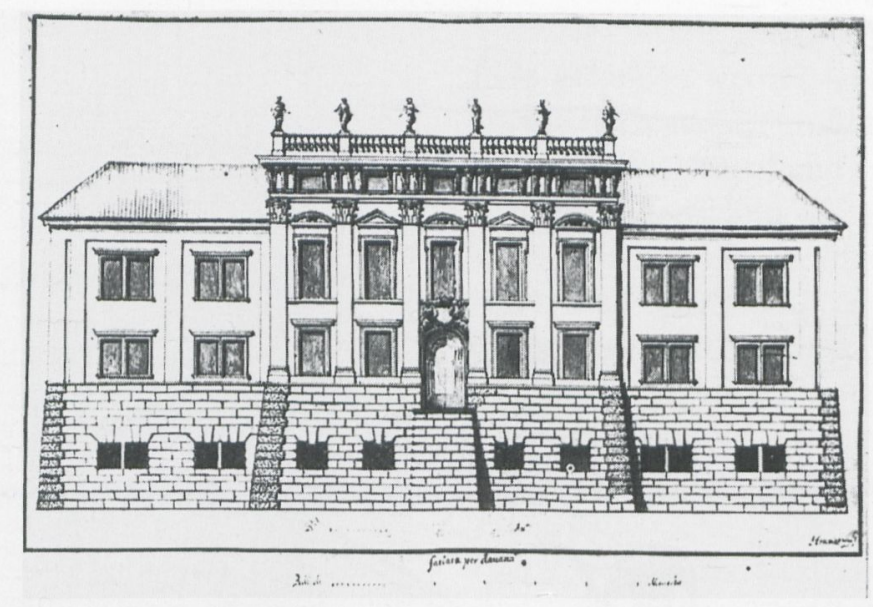

13. E. Zuccalli, Fassadenriß für Schloß Austerlitz (Slavkov), Mähren, Mailand, Castello Sforzesco, Racc. Martinelli, II,2

die Formen der Fenstereinfassungen im Piano nobile mit ihren alternierenden Verdachungen, die allgemein seit der ersten Hälfte des I6. Jahrhunderts an römischen Palästen, vor allem an den Werken von Antonio da Sangallo d. J., oder an späteren Bauten auftreten, im Werke von Domenico Fontana und Lorenzo Bernini. Sogar die ovalen Mezzaninfenster im Gebälkfries, wie sie die erste der beiden hier zu erörternden Zeichnungen vorsieht, treffen wir nicht selten in der römischen Architektur, um nur an das Collegio Romano der Jesuiten zu erinnern (I583, B. Ammanati und G. Valeriani) oder an die Flügel des Palazzo Barberini (ab 1628, C. Maderno und G. L. Bernini) ${ }^{21}$.

Der Autor der hier behandelten Gruppe von Entwurfsstudien muß also unter den italienischen Architekten gesucht werden, die die Architektur

${ }^{21}$ Solche Fenster erscheinen bereits im Entwurf des Palastes Ferrari-Casa del Pozzo (um I526, Antonio da Sangallo d. J.), siehe Christoph Luitpold Frommel: Der Römische Palastbau der Hochrenaissance, Tübingen 1973, II, 176, III, Taf. 7I-a.

${ }^{22}$ Siehe Richard A. L. Paulus: Der Baumeister Henrico Zuccalli am kurbayerischen Hofe zu München, Straßburg 1912; Sabine Heym: Henrico Zuccalli (um I6421724). Der kurbayerische Hofbaumeister, ZürichMünchen 1984 .

${ }^{23}$ Heym (wie Anm. 22), 23-32, I0I-I02; Lorenz (wie Anm. 7), 642-647.
Roms kannten und besonders von Berninis Entwürfen fasziniert waren, und im Kreise derer, deren künstlerische Kontakte bzw. Lebenswege sie in die Länder Mitteleuropas geführt haben. Unter den hier näher in Betracht kommenden Künstlern verdient der in der Schweiz geborene, in Rom ausgebildete und seit den siebziger Jahren in Bayern und Österreich tätige Enrico Zuccalli (um 1642 - 1724) besondere Beachtung ${ }^{22}$. Schon in seinen ganz frühen Werken und Entwürfen knüpfte dieser Architekt sowohl im Bereich der Sakral- wie auch der Profanbauten direkt an Berninis Kunst $\mathrm{an}^{23}$. Seine für Graf Dominik Andreas Kaunitz in den Jahren 1687 - I688 ausgeführten Pläne für den Umbau des Schlosses Austerlitz in Mähren (Sławków bei Brünn) sowie seine Projekte für das neue Stadtpalais in Wien (das spätere Palais Liech-

${ }^{24}$ Erich Hubala: Das Schloß Austerlitz in Südmähren, in: Adalbert-Stifter-Jahrbuch, V, 1957, 174-200, Abb. XIII, XV, XVI; Hellmut Lorenz: Enrico Zuccallis Projekt für den Wiener Stadt-Palast Kaunitz-Liechtenstein, in: Österreichische Zeitschrift für Kunst und Denkmalpflege, XXXIV, 1980, I6-22, Abb. 20, 22, 25; idem: Domenico Martinellis Projekt für Schloß Austerlitz (Slavkov u Brna) in Mähren, in: Umêní, XXIXXXXI, 198I, 250-25I, Abb. I-2; idem: Liechtenstein Palaces in Vienna from the Age of the Baroque, New York 1985, II-I6; idem: Domenico Martinelli und die österreichische Barockarchitektur, Wien 199I, 33-39, 


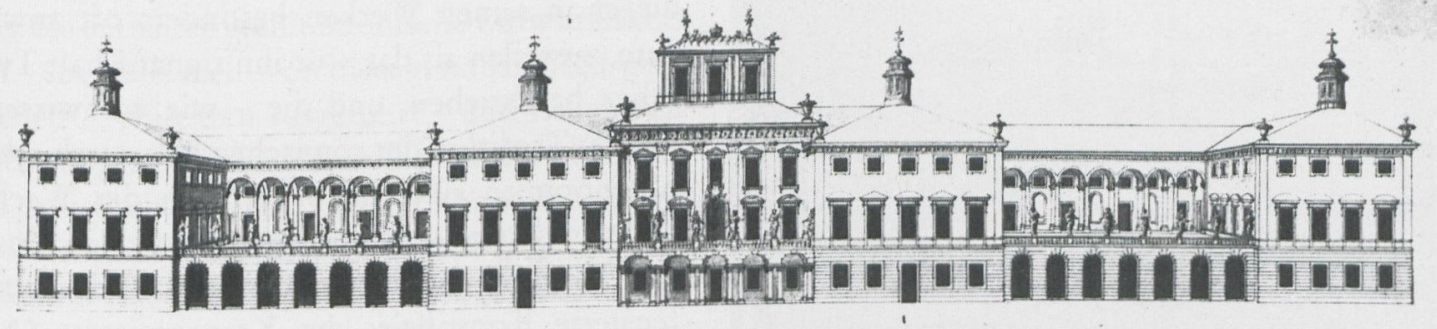

I4. E. Zuccalli, Entwurf zum Schloß Schleißheim, München, BHSTA, Nr. 8257 - Fragment

tenstein) führten in die Architektur der habsburgischen Länder einen Fassadentypus ein, der Berninis Palazzo Chigi-Odescalchi zum Vorbild hatte. Und dieser Typus bildete von da an das klassische Modell für die Fassaden von Stadtpalästen der berühmtesten österreichischen Architekten Johann Bernhard Fischer von Erlach und Lukas von Hildebrand ${ }^{24}$. Zuccalli war sein ganzes Leben hindurch von Berninis erstem Louvre-Projekt fasziniert. Als Hofarchitekt des Kurfürsten Max II. Emanuel von Bayern (1680 - 1726) seit Beginn der neunziger Jahre mit dem Entwurf für dessen Hauptresidenz in Schleißheim bei München betraut, hatte er in einem frühen Stadium seiner langjährigen Entwurfsarbeiten Lösungen vorgeschlagen, die direkt auf Berninis Konzeption basierten. Eine der Lösungen war die verdoppelte Wieder-

I54-I55, 227-228, Abb. 33, 8r; Heym (wie Anm. 22), 7I-75. ${ }^{25}$ München, Bayerisches Hauptstaatsarchiv, Plansammlung, Nr. 8282 (andere: Nr. 8270, 8280). Vgl. Erich Hubala: Schleißheim und Schönbrunn, in: Kunstchronik, X, 1957, 349, 353, Abb. 2-3; Michael Petzet: Unbekannte Zuccallis für die Schleißheimer Schloßbauten, in: Münchner Jahrbuch der Bildenden Kunst, 1971, 189-190, Anm. 35 und 39, Abb. I5; Dorith Riedl: Henrico Zuccalli. Planung und Bau des neuen Schlosses Schleißheim, München 1977, 6; Lorenz: "Lustgartengebäude « I, 7I-72, Abb. 80; Heym (wie Anm. 22), 56-58. holung dieses Vorbildes ${ }^{25}$. Nach vielen Jahren kehrte Zuccalli zu letzterem in seinem Entwurf für die Fassade der Benediktinerkirche in Ettal (ab 1709) zurück ${ }^{26}$.

Die Vermutung, der Autor der zu erörternden Warschauer Zeichnungen sei Zuccalli, wird auch durch die Ähnlichkeiten bestätigt, die zwischen den Einzelheiten der Skizzen und den Werken und Ausführungsprojekten des bayerischen Architekten sichtbar werden. Die verwandte Komposition eines Baukörpers mit eingezogenem, mit kolossaler bzw. großer Pilasterordnung instrumentiertem Mittelteil finden wir denn auch in dem von Zuccalli entworfenen Schloß Lustheim (16841687) wieder sowie in einigen Entwürfen für das neue Schloß Schleißheim (zwischen 1693 und I70I, Abb. 14$)^{27}$. Auch die Arkaden-Loggia, eingebun-

${ }^{26}$ Heym (wie Anm. 22), 88-98.

${ }^{27}$ Lustheim siehe: Petzet: (wie Anm. 25), 179-182; Gerhard Hojer: Die Münchner Residenzen des Kurfürsten Max Emanuel, in: Kurfürst Max Emanuel. Bayern und Europa um 1700, Bd. I, München 1976, I44-146; Heym (wie Anm. 22), 42-50. Es muß betont werden, daß die Seitenfassaden des Schlosses Lustheim analog zu den Projekten für das »Lustgartengebäude « gelöst wurden (siehe Lorenz: "Lustgartengebäude« I, 72, Abb. 82). Projekte für das Schloß Schleißheim - BHSTA Nrn. 8257, 826I, $828 \mathrm{I}$ - siehe Petzet (wie Anm. 25), I87-I89, Abb. I2-I4; Riedl (wie Anm. 25), 6; Heym (wie Anm. 22), 55-57. 


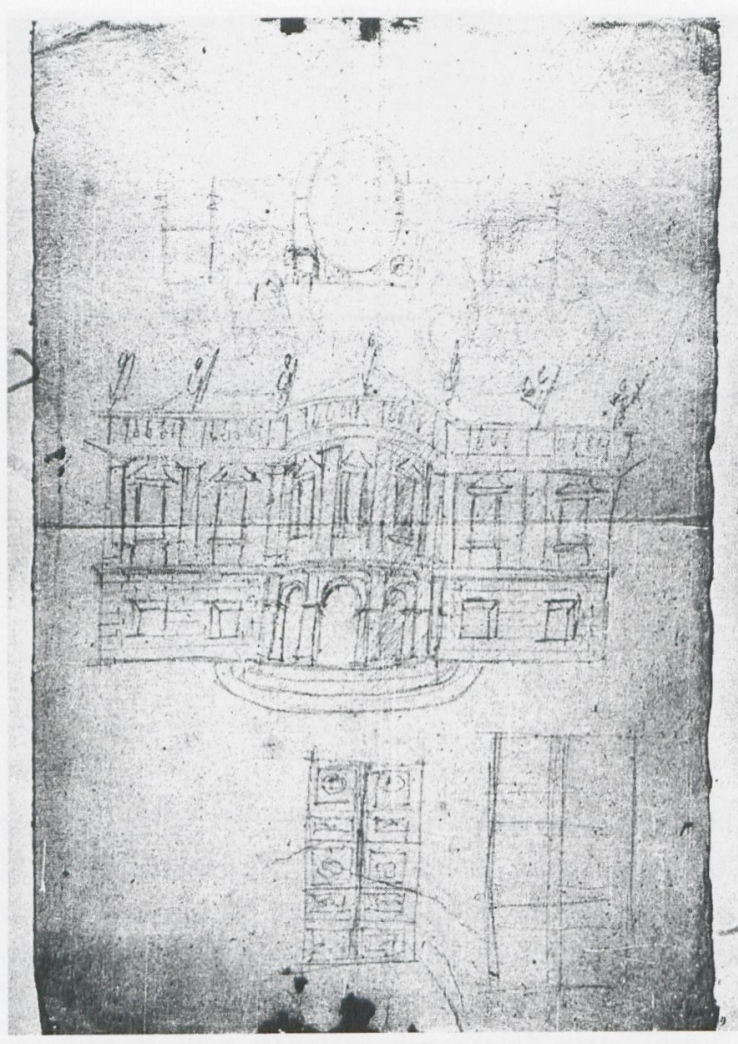

16. Entwurfskizzen zum Gartenpalais »Belvedere« in Warschau, Warschau, Univ.-Bibl., AT 454 recto

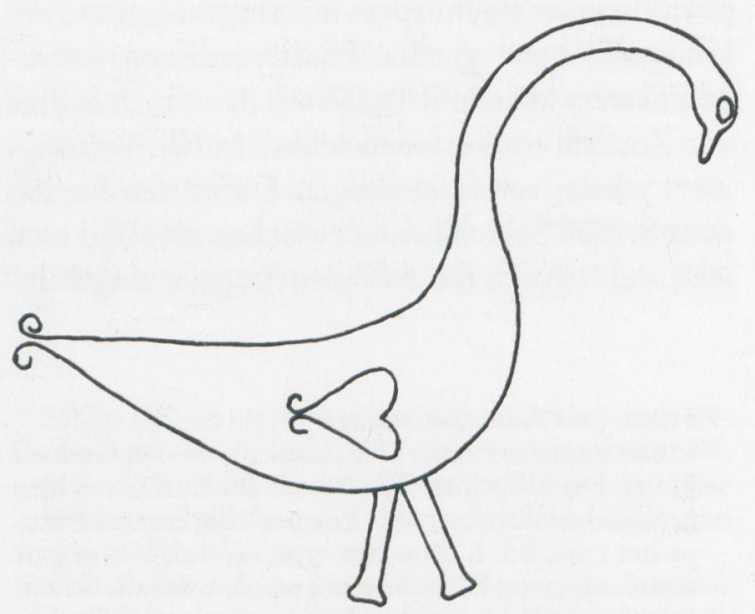

I5. Wasserzeichen von Blatt AT 453, AT 479 den zwischen Pilaster großer Ordnung, gehörte zu den Kompositionen, die der Schöpfer der bayerischen Residenz schätzte ${ }^{28}$. Zu den Motiven, die er in seinen Werken besonders oft anwendete, zuweilen als das »für ihn signaturhaft Typische" beschrieben, und die - wie wir wissen aus der Tradition der römischen Palastarchitektur übernommen sind, gehörten auch der Wechsel von gerade und segmentbogenförmig geschlossenen Fenstereinfassungen und ebenfalls der durchfensterte Konsolfries des Kranzgesimses (Abb. I3) ${ }^{29}$. Auch war dem Künstler die römische Art, Fenster von Erdgeschoß und Sockelzone zusammen einzufassen, nicht fremd, und er kannte das »Herausschneiden « der ovalen Mezzaninfenster aus dem Gebälkfries; letztgenanntes Motiv verwendete er in beiden Fassaden von Schleißheim 30 .

Die publizierten Entwürfe Zuccallis sind in der Regel sorgfältig ausgearbeitete Zeichnungen möglicherweise sogar unter Beteiligung seiner Mitarbeiter entstanden -, infolgedessen ist es schwer, sie in Bezug auf Einzelheiten in der Ausführung, der Linienführung u. ä. mit den in Warschau aufbewahrten Skizzen zu vergleichen. Um so mehr sind einige stilistische Elemente, wie z.B. das Abtönen der Fensteröffnungen und der Loggien-Durchblicke durch Striche bzw. Lavierungen, den bekannten Entwürfen des bayerischen Architekten und den Zeichnungen in Tilmans Sammlung gemeinsam.

Wenn unsere Vermutung richtig ist und die hier erörterten Zeichnungen von Zuccalli geschaffen worden sind, stellen sich folgende Fragen: woher stammen die Zeichnungen, wie gelangten sie in

\footnotetext{
${ }^{28}$ Projekte BHSTA Nr. 825I, 8249 und Bayerische Verwaltung der Staatlichen Schlösser und Gärten, Bauamt - siehe Erich Hubala: Henrico Zuccallis Schloßbau in Schleißheim. Planung und Baugeschichte $1700-1704$, in: Münchner Jahrbuch der Bildenden Kunst, XVII, I966, 166, 168-169, 184-189, 194, 196, Abb. 21, 19, 20; Riedl (wie Anm. 25), 8; Lorenz: »Lustgartengebäude $\mathrm{I}, 71, \mathrm{Abb}$. $8 \mathrm{I}$.

${ }^{29}$ Siehe Schloß Lustheim, Entwürfe für das Schloß Austerlitz, Palais Kaunitz-Liechtenstein in Wien und Schloß Schleißheim (BHSTA Nr. 8262 - Hubala: wie Anm. 28, 166, 168-169, Abb. 9).
} 
Tilmans Hände und wann sind sie entstanden? Bei ihrer Beantwortung helfen meiner Meinung nach vier weitere Blätter aus dem Nachlaß van Gamerens: Sie enthalten architektonische Entwurfsskizzen - ebenfalls nicht von diesem holländisch-polnischen Architekten ausgeführt - und stehen den Zeichnungen, die wir Zuccalli zugeschrieben haben, stilistisch auffallend nahe. Es handelt sich um die Zeichnungen auf den Blättern AT 454, AT 453 recto und verso, $\mathrm{AT}_{455}$ und $\mathrm{AT} 479$ (Abb. $16-20$ ), wobei das erste und das dritte Blatt keine Wasserzeichen haben. Die anderen beiden sind mit einem Zeichen versehen, das einen stilisierten Schwan im Profil zeigt (Abb. 15) ${ }^{3 \text {. }}$. Wir haben es hier mit mehr oder weniger skizzenhaften Entwurfsphasen zu ein und demselben zweigeschossigen Bau vom Typus Gartenpalais zu tun. Instrumentiert mit großer Pilasterordnung über rustiziertem Sockel und bekrönt von einer Balustradenattika mit Statuen birgt dieser Bau in der Mitte einen ovalen, risalierten Saal, der vom Hof wie vom Garten her durch Freitreppen zugänglich ist. Die kompositionelle Lösung für den Saal ist das Hauptthema der Skizzen auf den Blättern AT 454 und AT 453 recto und verso (Abb. I6-I8). Anfänglich rechtekkig und an der Rückfassade risalitartig hervorgeschoben, wird der Saal danach zu einem ovalen Innenraum, der aus der Flucht an beiden Fassaden heraussteht, und dann endgültig nur an einer Seite halbkreisförmig hervortritt. Die Veränderungen dieses Raumes werden durch aufeinanderfolgende Varianten zur Gestaltung des Baukörpers und zur Komposition der Außentreppen begleitet, die ebenfalls auf den erwähnten Blättern dargestellt sind.

${ }^{30}$ Für die Verbindung von Fenstereinfassungen siehe den Entwurf für das Palais Kaunitz-Liechtenstein und das frühe Projekt für das Schloß Schleißheim (BHSTA Nr. 8253 - Petzet: wie Anm. 25, 186-187, Abb. II). Die Mezzaninfenster wurden in gerader dieser Form realisiert.

${ }^{31}$ Die drei ersten stellen Bleistiftzeichnungen dar, die Linien der letzteren wurden mit Feder nachgezogen. Maße der Zeichnungen: AT 454-325 x 2I2 mm; AT 453$416 \times 321 \mathrm{~mm}$; AT 455-210 × $277 \mathrm{~mm}$; AT 479-4I4 X 320 mm. Das auf den Zeichnungen AT 453 und 479 sichtbare Wasserzeichen konnte keiner Papierfabrik zugeordnet werden. Zeichnung AT 479 wurde (mit fal-

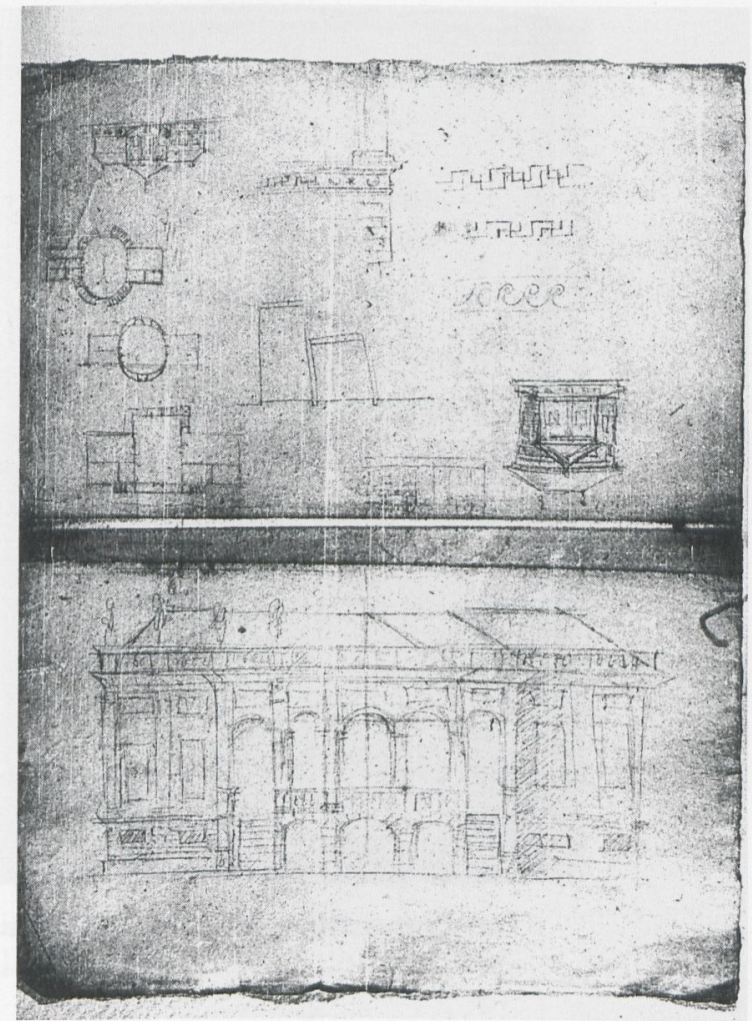

17. Entwurfskizzen zum Gartenpalais »Belvedere« in Warschau, Warschau, Univ.-Bibl., AT 453 recto

Daß der Entwurf auf dem Blatt AT 454 (Abb. 16), der einen höheren Sockel vorsieht, durch den eine ovale, nach außen in einer Arkaden-Loggia geöffnete Sala Terrena unter dem Saal eingeführt werden kann, gleichsam ein Verbindungsglied zwischen dieser Serie von Zeichnungen und den besprochenen Projekten für Bauten vom Typus »Lustgarten-

scher Signatur) veröffentlicht von Jolanta Putkowska: Rezydencja w Ujazdowie w drugiej połowie XVI i w XVII wieku, czȩść II. Rezydencja Stanisława Herakliusza Lubomirskiego (Residenz in Ujazdów in der zweiten Hälfte des I6. und im I7. Jh., 2. Teil. Residenz Stanisław Herakliusz Lubormirskis), in: Kwartalnik Architektury i Urbanistyki, XXII, 3, 1977, 195-196, Abb. 3, die sie unzutreffend mit den auf das Łazienki-Palais sich beziehenden Projekten Tilmans van Gameren in Verbindung gebracht hat (u. a. mit der Zeichnung AT 480, die einen fast zwei Drittel kleineren Pavillon darstellt). 


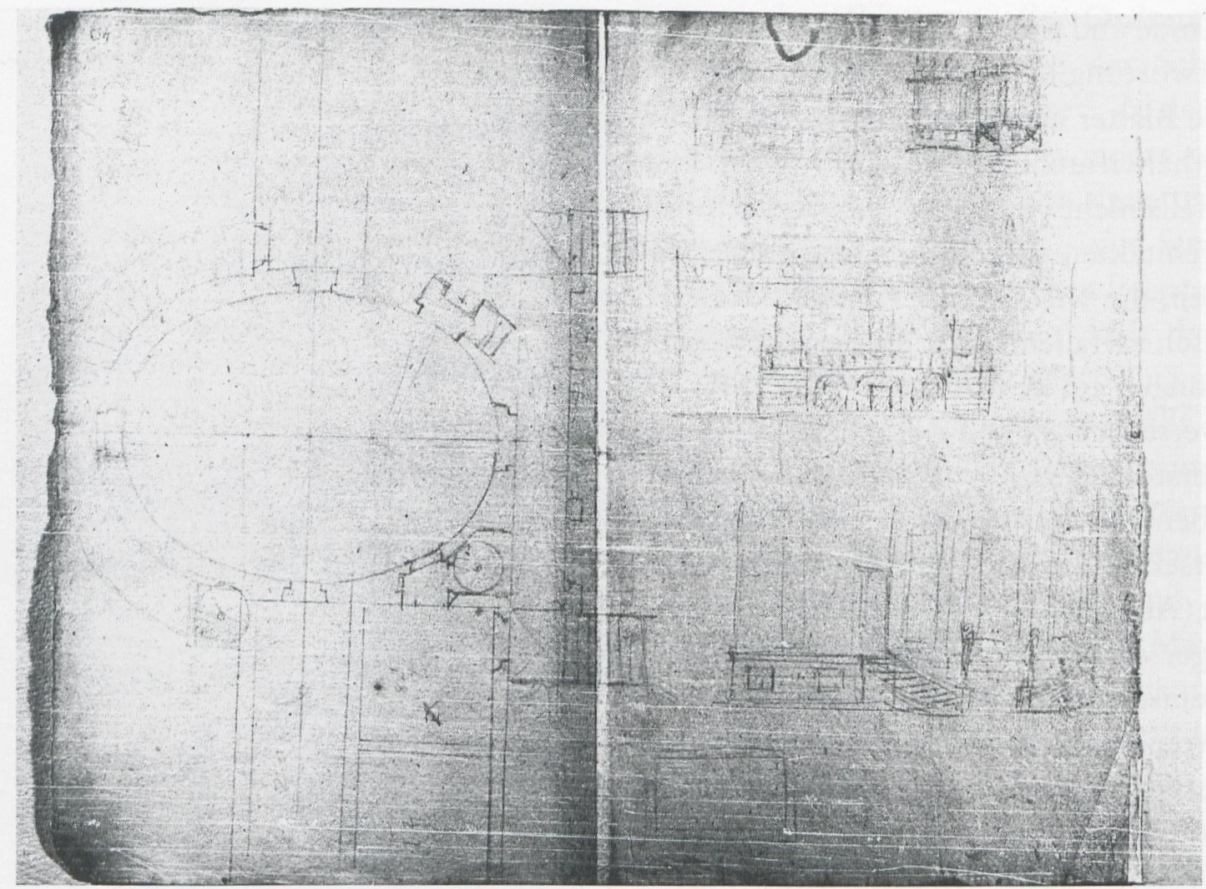

I8. Entwurfskizzen zum Gartenpalais »Belvedere« in Warschau, Warschau, Univ.-Bibl., AT 453 verso

gebäude« bildet, ist nicht zu übersehen. Die Skizzen auf den übrigen drei Blättern bezeugen hingegen, daß der Künstler die Idee des hohen Sockelgeschosses mit Sala terrena zugunsten eines vorteilhafteren Piano nobile als hochgelegenes Erdgeschoß verworfen hatte. An der Gartenfassade sollen zwei, auf symmetrisch angelegte Eingänge zulaufende Treppenarme den oval herausgeschobenen Saal einfassen, eine Arkaden-Loggia mit einem Paar gerader Treppenläufe hat das Risalit an der Vorderfassade zu bilden. Der Ausführungsentwurf erscheint auf der Zeichnung AT 455 (Abb. 19); die Gesamtanlage mit den Hofbauten, eingeschrieben in ein genau vermessenes Grundstück, bringt die Zeichnung AT 479 (Abb. 20).

Der Formenapparat, der in den verschiedenen Entwurfsphasen zur Anwendung kommt - darunter auch das Motiv der oval auseinanderlaufen-

${ }^{32}$ Außentreppen - vgl. das Projekt für Schloß Schleißheim BHSTA Nr. 826r und 828ז (Petzet: wie Anm. 25, I87-188, Abb. I2-13). den Treppen - besitzt nahe Analogien zu den Werken und Entwürfen von Zuccalli32 ${ }^{32}$. Besonders auffällig ist die formal-stilitische Ähnlichkeit seiner Entwurfszeichnungen mit den Warschauer Skizzen: Sie ist in solchen Elementen sichtbar wie den schraffierten Tür- und Fensteröffnungen, den teilweise "pseudoperspektivisch « angegebenen Details - z. B. den Ausschnitten der Erdgeschoßfenster auf der Zeichnung AT 545 und den Gebälkkonsolen auf der Zeichnung AT 455-und schließlich in den auf charakteristische Art skizzierten Attika-Figuren und ihren Posen auf der Zeichnung AT $455^{33}$. All das zusammen läßt es meiner Meinung nach zu, auch diese Gruppe von Zeichnungen mit Enrico Zuccallis Schaffen zu verbinden. Ein zusätzliches Argument für die Autorschaft des kurfürstlich-bayerischen Architekten sind schließlich Skizzen für ein dekoratives Mo-

33 »Pseudoperspektivische« Darstellung der Details (z. B. der Konsolen des Gebälkfrieses) finden wir u. a. in den Fassadenentwürfen für das Schloß Austerlitz und das 


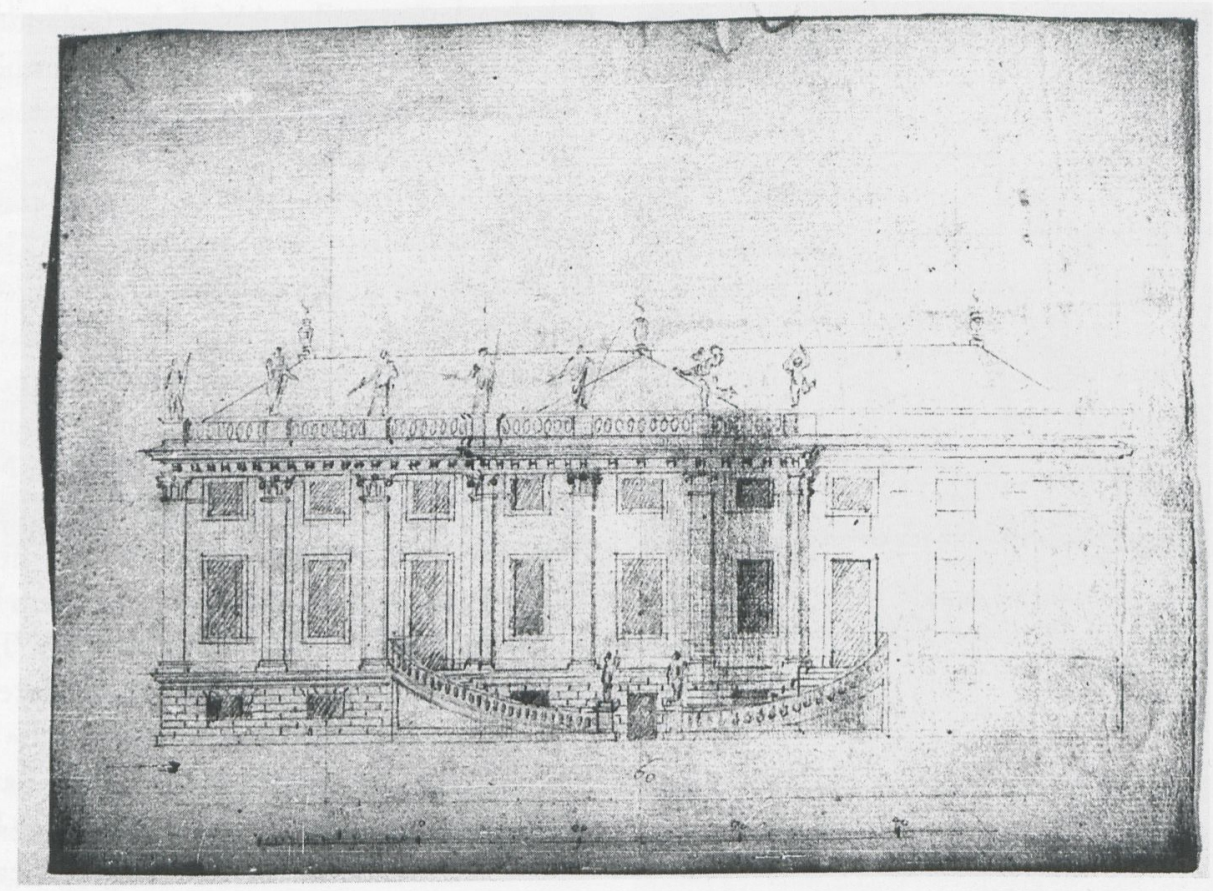

19. Entwurf der Gartenseite des Warschauer »Belvedere«, Warschau, Univ., Bibl., AT 455

nogramm auf der Rückseite des Blattes AT 454, wo wir in den einfallsreichen Geflechten die Buchstaben $» M \ll$ und $» X$ « erkennen, gleichsam die Abkürzung des Namens Max.

Die zuletzt besprochene Gruppe von Zeichnungen enthält auch wichtige Informationen für eine eventuelle Identifizierung des Entwurfs, so z. B. eine Skala, durch die wir die Maße des Baues und den Grundriß der ganzen Parzelle erfahren können (Zeichnungen AT 455 und AT 479, Abb. 19, 20). In die nicht ebenmäßige, an einer Ecke schräge Form des Grundstücks ist hier eine rechteckige Anlage mit den Maßen I 51 x I20 Ellen (ca. 90 x 71 m) eingeschrieben. In der Tiefe direkt am Rand des Grundstücks sollte der uns bekannte Bau mit einer Fassadenbreite von 60 Ellen (ca. $36 \mathrm{~m}$ ) stehen. An diesem Punkt muß daran erinnert werden, daß Tilman van Gameren im Prinzip weder rein theo-

Palais Kaunitz-Liechtenstein, und die Figur mit dem charakteristisch gehobenen Bein bildet den Abschluß eines der Pavillons aus dem Projekt des Schlosses retische Entwürfe noch Arbeiten anderer Architekten als Muster zur Nachahmung sammelte. Bei den nicht sehr zahlreichen Zeichnungen von fremder Hand in seinem Archiv handelte es sich in der Regel um mit einem konkreten Bau verbundene Studien, anhand derer er ebenfalls später eigene Arbeiten ausführte. Daher stellen auch die angegebenen genauen Maße und die Form des Grundstücks nicht zu unterschätzende Informationen dar, um so mehr, als ein einen ähnlich gelegenen Bau vom Typus Gartenpalais in der nächsten Nachbarschaft der Residenz Ujazdów bei Warschau wiederfinden: letztere gehörte dem Hauptauftraggeber van Gamerens, dem Fürsten Stanisław Herakliusz Lubomirski. Ich denke bei diesem Bau an das später berühmte Belvedere, das in der zweiten Hälfte des 17. Jahrhunderts im Besitz der litauischen Familie Pac war. Aus dieser

Schleißheim - BHSTA Nr. 8252 (Petzet: wie Anm. 25, I86-I87, Abb. I0). 


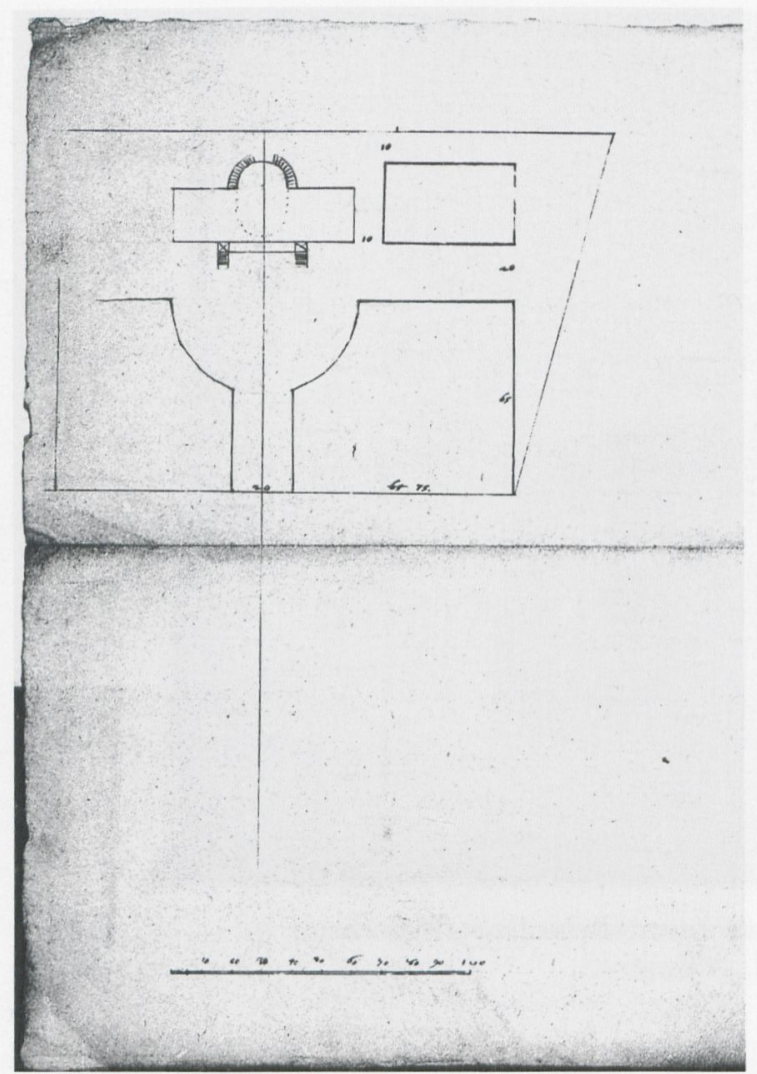

20. Plan des Gartenpalais »Belvedere« in Warschau, Warschau, Univ.-Bibl., AT 479

Zeit sind uns keinerlei Bildmaterialien erhalten. Wir kennen die Form und die Bebauung der Belvedere-Parzellen einzig aus den Vermessungen der Ujazdów-Güter; die älteste ist erst in der Zeit zwischen 1720 und 1733 durchgeführt worden (Abb. 21, 22) ${ }^{34}$. Die mit denen auf der Zeichnung AT 479 fast gleichen Maße des nahezu quadratischen Grundstückes (Breite ca. I5o Ellen, d. s. ungefähr $90 \mathrm{~m}$ ) fallen hier ebenso auf wie die Lage des Pavillons am hinteren Rand der Parzelle, was

${ }^{34}$ Dresden, Sächsisches Landeshaupt-Archiv, Sign. VII, 90, Nr. 35b-d; Sign. Sächsisches Landesamt für Denkmalpflege Archiv M. 69.X.Lh. 13; VII, 89, Nr. 3, Blatt i (Situationsplan des auf den Gebieten von Ujazdów geplanten Kalvarienweges, ausgearbeitet von J. J. D. Jauch im Jahre 173I). Siehe: Varsaviana w zbiorach drezdeńskich. Katalog (Varsaviana in den Dresdner Sammlungen. Katalog), Warschau 1965, 77-78, Pos. 191, sich durch den steilen Abfall des Geländes an dieser Seite erklärt ${ }^{35}$. Diese außergewöhnliche Aussichtsposition hatte letzten Endes den Namen »Belvedere « bedingt.

Dank der Forschungen u. a. von Marek Kwiatkowski ist bekannt, daß der litauische Großkanzler Krzysztof Zygmunt Pac auf dem hier 1659 erworbenen Grundstück ein »Herrenhaus « genanntes Gebäude sicher als Holzbau hatte errichten lassen. Dieses überschrieb er 1663 seiner Ehefrau Klara Izabella, geb. de Mailly Lascaris. Nach dem Tod des Kanzlers - er starb am Io. Januar 1684 im Belvedere - vermachte seine Witwe nun diesen Besitz ihrem angenommenen Sohn, dem MalteserRitter Kazimierz Michał Pac. In dem Testament aus dem Jahre 1685 wird das Belvedere »Palais « genannt und sein Garten als »italienisch « beschrieben. Ob es sich um denselben kleinen Bau handelte, dessen quadratischer Umriß ( $40 \times 40$ Ellen) auf den oben erwähnten Vermessungsplänen aus dem 18. Jahrhundert zu sehen ist, ist nicht bekannt. Auf jeden Fall wurde das damalige Belvedere von Kazimierz Michał Pac 1697 an Katarzyna de Landrovie Michałowska verkauft, und, nachdem es so oft verpachtet war und den Besitzer gewechselt hatte, in den dreißiger Jahren des I8. Jahrhunderts (vor 1739) durch einen Bau nach Plänen des Architekten Józef Fontana ersetzt ${ }^{36}$.

Wenn unsere Annahme richtig ist und sich die Gruppe der besprochenen Entwürfe tatsächlich auf das bei Warschau gelegene Belvedere bezieht, stellt sich die Frage, wer die Projekte bei dem italienisch-bayerischen Architekten in Auftrag gegeben haben kann und wann dies geschah. Als die Familie Pac das Anwesen besaß und vor allem in der Zeit des letztgenannten Kazimierz Michał, wurde der zwischen Warschau und der der Hauptstadt benachbarten königlichen Residenz

193, 219; Putkowska (wie Anm. 31), 198, 194, Abb. 6, 2. ${ }^{35}$ Die schräge Erweiterung an der rechten Seite des Grundstückes auf der Zeichnung AT 479 findet ihre Bestätigung in der auf dem Plan von Jauch (SLA VII, 89, Nr. 3, Blatt I, Abb. 22) eingezeichneten Baumreihe, die das damals zur Kirche gehörende Gelände schräg durchschneidet und vermutlich die frühere Grenze zwischen den Parzellen markiert. 


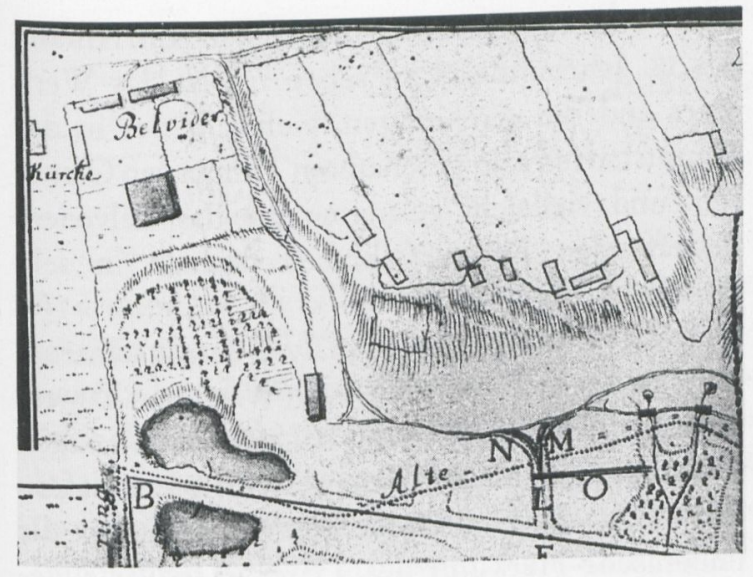

2I. Plan der »Belvedere«-Anlagen, Dresden, Sächsisches Landeshaupt-Archiv, VII, 90, Nr. 35 b-d

Wilanów so schön auf der Anhöhe gelegene Bau ziemlich häufig durch den Hof von König Johann III. Sobieski genutzt. Bereits 1677 wohnten hier die kurländischen Gesandten, als sie dem neugewählten Monarchen zu huldigen in die polnische Hauptstadt gekommen waren ${ }^{37}$. Es ist auch bekannt, daß Johann III. am 20. Mai 1690 feierlich im Belvedere empfangen worden ist ${ }^{38}$. Als nächstes hielt sich hier im März I69ı die Prinzessin Hedwig Elisabeth aus der Neuburger Linie der Wittelsbacher, Tochter des Pfalzgrafen von Neuburg, Philipp Wilhelm, vor ihrer Hochzeit mit dem königlichen Prinzen Jakub Sobieski auf. Hier wurde sie von ihrem Verlobten willkommen geheißen und vom Königspaar incognito besucht ${ }^{39}$. Daher scheint es ziemlich wahrscheinlich, daß in den neunziger Jahren der Gedanke aufgekommen sein könnte, das Belvedere vom Malteser-Ritter für die Belange der Familie und des Hofes von Johann III. zu pachten, und daß gleichzeitig die ganz natürliche Idee entstand, das Gebäude entsprechend um-

${ }^{36}$ Siehe: Marek Kwiatkowski: Niechaj twych ulic wiatr mnie owionie. Architektura warszawskich dzielnic, Warschau 1973, 38-42, Abb. 22-26; idem: Belweder (Zabytki Warszawy), Warschau 1976, 6-16, Abb. 1-4. Zum Tod des Kanzlers Pac siehe: Halina KairiūkštytéJacyniené: Pažaislis, ein Barockkloster in Litauen, Kaunas 1930,38 .

37 Kwiatkowski (wie Anm. 36, 1973), 4I.

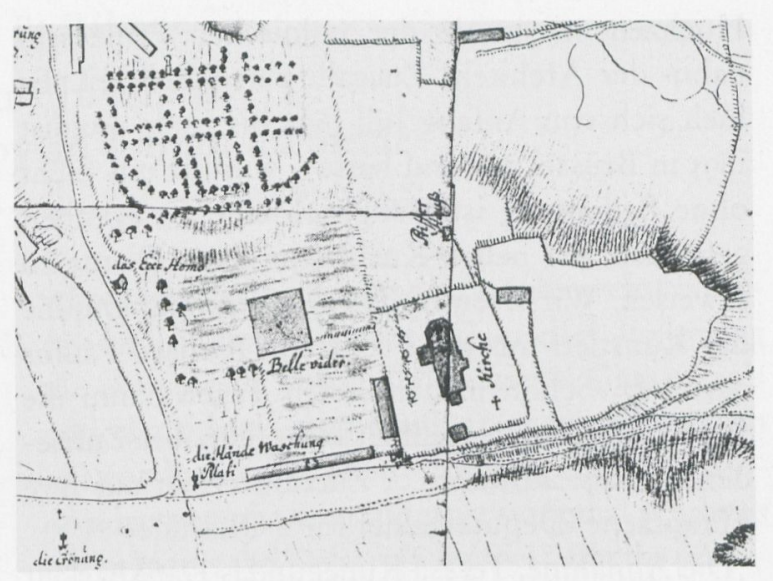

22. J. D. Jauch, Plan der »Belvedere«-Anlagen, Dresden, SLHA VII, 89, Nr. 3, Blatt I

bauen und modernisieren zu lassen. Kazimierz Pac war offensichtlich nicht allzu sehr mit seinem Sitz bei Warschau verbunden, da er ihn einige Jahre später verkaufte. Dazu ergab sich durch eine andere Ehe innerhalb der königlichen Familie eine vortreffliche Gelegenheit, das Talent des Hofarchitekten der bayerischen Wittelsbacher in Anspruch zu nehmen. Ich denke hier an die im Mai 1694 in Żółkiew beschlossene und zweimal - per procuram am 19. August 1694 in Warschau und dann am 2. Januar 1695 in Brüssel feierlich - geschlossene Ehe der königlichen Prinzessin Teresa Kunegunda Sobieska mit Max II. Emanuel, dem bayerischen Kurfürsten und Statthalter der Niederlande, dem Hauptauftraggeber von Zuccalli ${ }^{40}$. Es muß hier erwähnt werden, daß der neue Schwiegersohn dem polnischen König als Mitstreiter beim Entsatz von Wien schon lange bekannt war; dort hatte sich Max Emanuel unter Sobieskis Führung seine ersten militärischen Verdienste erworben. An der eigentlichen festlichen

${ }^{38}$ Putkowska (wie Anm. 31), 199, Anm. 37.

39 W. Kulczycki: Djariusz podróży do Polski wyjęty z pamiętników Jana Chrzciciela Fagiuoli (Tagebuch der Reise nach Polen, entnommen den Erinnerungen des Giovanni Battista Fagiuoli), Beilage zu: Czas, Krakau, August I858, 287.

$4^{\circ}$ Siehe u. a. Kurfürst Max Emanuel. Bayern und Europa um I700, Ausst.-Kat., München 1976, II, II5, 126. 
Hochzeitszeremonie der polnischen Prinzessin nahm der Architekt Zuccalli persönlich teil. Er hielt sich von Anfang Juli 1694 bis Ende August I695 in Brüssel auf und hatte - was für uns nicht ohne Bedeutung ist - sogleich eine Gelegenheit gefunden, der neuen Kurfürstin einen Dienst zu erweisen. Wir lesen in der Quellenmonographie des Künstlers aus der Feder von Richard Paulus darüber: "Schon in den ersten Tagen nahm die Kurfürstin die Gelegenheit wahr, ihm ihre Zufriedenheit auszudrücken... Zuccalli erhält auf ihre Fürsprache $>$ Deputatgelder für 2 Quartale« « 4 .

Die Annahme, Teresa Kunegundas Dankbarkeit sei u. a. mit der Anfertigung von Entwürfen für das Belvedere bei Warschau verbunden gewesen, und, was sehr wahrscheinlich ist, nicht nur mit den Arbeiten zur Ausstattung der Hochzeitsfeierlichkeiten, geht wohl nicht zu weit. Wenn sich die Gruppe der Warschauer Zeichnungen also wirklich auf das Belvedere bezieht und aus Enrico Zuccallis Werkstatt stammt, muß man sie in jedem Fall auf die Zeit nach der Verlobung Teresa Kunegundas 1694 und vor dem Tod ihres Vaters König Johann III. am 17. Juni 1696 datieren, also in den Zeitraum, der als Höhepunkt im Schaffen des bayerischen Architekten anerkannt ist ${ }^{42}$.

Tatsache ist jedoch, daß die hier besprochenen, bisher nicht zugeordneten Entwürfe in Warschau nicht realisiert worden sind. Der kleine quadrati-

${ }_{41}^{4}$ Paulus (wie Anm. 22), 115; sowie Anm. 200.

${ }^{42} \mathrm{Heym}$ (wie Anm. 22), 18.

${ }^{43}$ In diesem Kontext fällt eine Übereinstimmung der Entwürfe zum Schloß Lützenburg (Charlottenburg) bei Berlin (1695-1699) auf, das dem anderswo wenig bekannten Architekten Johann Arnold Nering (gest. am 2I. I0. 1695) zugeschrieben wird, mit dem Entwurf zu dem Bau auf den oben besprochenen Zeichnungen AT 452 und 453 . Der Bau bei Berlin erscheint wie eine Verschmelzung der auf diesen beiden Zeichnungen sichtbaren Projekte (Abb. 8, I8). Es ist auch bekannt, daß der Entwurf für das Schloß der Ehefrau des Kurfürsten Friedrich III. nach langwierigen Konsultationen entstanden ist; im Jahre 1694 wurde es auf Vermitt- sche Pavillon, welcher auf den Plänen vom Anfang des I8. Jahrhunderts zu sehen ist, ist das Werk eines anderen Architekten, vielleicht des in der Hauptstadt so sehr geschätzten Tilman van Gameren: und vielleicht gelangten die das Belvedere betreffenden Entwurfsskizzen deshalb in sein Archiv.

Die hier durchgeführte Analyse der rätselhaften Zeichnungen, die vor 1706 nach Warschau kamen, veranlaßt meiner Meinung nach auch zu einigen Feststellungen allgemeinerer Art. Erstens liefern diese Blätter ein zusätzliches Argument für die italienische Herkunft des Typus "Lustgartengebäude «, der traditionell mit Johann Bernhard Fischer von Erlach assoziiert wird, und sie scheinen die These zu erschüttern, der österreichische Meister habe Zuccalis Schaffen beeinflußt. Zweitens zeigen die Zeichnungen in vorzüglicher Weise die Internationalität der mitteleuropäischen Architektur um 1700 und den stetigen Austausch künstlerischer Werte zwischen den einzelnen Zentren ${ }^{43}$. Und schließlich, drittens, machen die Zeichnungen die besondere Rolle der italienischen Künstler in diesem Austausch sichtbar. Ihr Schaffen mit seinen Wirkungen an den Höfen - in Wien, wie in München, Warschau und Berlin -, war einer der Faktoren, die das gemeinsame Antlitz der Künstlerkultur in Mitteleuropa dieser Epoche bestimmten ${ }^{44}$.

lung des Grafen Nils Bielke nach Schweden geschickt, um die Meinung von Nicodemus Tessin d. Jg., des Hofarchitekten Karls XI., einzuholen (siehe Margarete Kühn: Schloß Charlottenburg, Berlin 1955, II-19, Abb. 4-6, 18; Ragnar Josephson: Tessin. Nicodemus Tessin d. Jg., Stockholm 1930, II, Abb. I47-I48; Goerd Peschken: Andreas Schlüter und das Schloß Charlottenburg, in: Schloß Charlottenburg, Berlin, Preußen. Festschrift für Margarete Kühn, München 1975, I4I-I47, Abb. 6, II, 19, 20).

44 Vgl. Hellmut Lorenz: Zur Internationalität der Wiener Barockarchitektur, in: Wien und der europäische $\mathrm{Ba}$ rock (Akten des XXV. Internationalen Kongresses für Kunstgeschichte), Wien 1986, 2I-30.

Abbildungsnachweis: I, 4, 6-8, IO-I2, I5-20 Archiv Autor. - 2 nach Sedlmayr. - 3 nach Brauer, Wittkower. 5, 13 nach Lorenz. - 9 nach Langenskiöld. - I4 BHSTA München. - 2I-22 Instytut Sztuki PAN. 